\title{
CONVERGENCE OF A GODUNOV SCHEME FOR CONSERVATION LAWS WITH A DISCONTINUOUS FLUX LACKING THE CROSSING CONDITION
}

\author{
K. H. KARLSEN ${ }^{\mathrm{A}}$ AND J. D. TOWERS ${ }^{\mathrm{B}}$
}

\begin{abstract}
We study a scalar conservation law whose flux has a single spatial discontinuity. There are many notions of (entropy) solution, the relevant concept being determined by the application. We focus on the so-called vanishing viscosity solution. We utilize a Kružkovtype entropy inequality which generalizes the one in [38, singles out the vanishing viscosity solution whether or not the crossing condition is satisfied, and has a discrete version satisfied by the Godunov variant of the finite difference scheme of [26, 38. We show that the solutions produced by that scheme converge to the unique vanishing viscosity solution. The scheme does not require a Riemann solver for the discontinuous flux problem. This makes its implementation simple even when the flux is multimodal, and there are multiple flux crossings.
\end{abstract}

\section{INTRODUCTION}

In recent years there have been many works on hyperbolic conservation laws with a spatially discontinuous flux function, providing a great number of results relating to existence, uniqueness, stability, and numerical approximations of entropy solutions [1, 2, 3, 4, 5, 3, 6, 7, 8, 9, 10, 11, 12, 13, 14, 15, 16, 17, 18, 19, 20, 21, 22, 23, 24, 25, 26, 27, 28, 29, 30, 31, 32, 34, 36, 37, 38, 39, 41, 42, 43, 44, 45, 46, 47. Herein we are interested in numerical methods for the initial value problem

$$
\begin{aligned}
u_{t}+\mathcal{F}(x, u)_{x} & =0 \quad \text { for }(x, t) \in \Pi_{T}:=\mathbb{R} \times(0, T), \\
u(x, 0) & =u_{0}(x) \quad \text { for } x \in \mathbb{R}, \\
\mathcal{F}(x, u) & :=H(-x) g(u)+H(x) f(u),
\end{aligned}
$$

where $H(x)$ is the Heaviside function. Thus, the flux $\mathcal{F}(x, u)$ of this conservation law has a spatial dependence that is discontinuous at $x=0$ if the functions $f$ and $g$ are different. We will assume that the initial data

$$
u_{0}(x) \text { lies in the interval } \mathcal{U}:=[\underline{u}, \bar{u}] .
$$

The flux functions satisfy

$$
f, g \in \operatorname{Lip}([\underline{u}, \bar{u}]),
$$

with Lipschitz constants $L_{f}$ and $L_{g}$. We need a condition that guarantees that the solution is bounded, which leads us to the following assumption:

$$
g(\underline{u})=f(\underline{u}), \quad g(\bar{u})=f(\bar{u}) .
$$

Assumption (1.4) appears in several applications, and has become a common assumption in the literature on conservation laws with discontinuous flux. Moreover, we assume that both $f$ and $g$ are genuinely nonlinear in the sense that

$$
f \text { and } g \text { are not linear on any non-degenerate interval. }
$$

We will need the following definitions:

Date: December 3, 2016

2010 Mathematics Subject Classification. Primary: 35L65, 65M06; Secondary: 65M06.

Key words and phrases. Conservation law, discontinuous flux, entropy solution, vanishing viscosity, adapted entropy, uniqueness, convergence, finite difference scheme, Godunov scheme, $\Gamma$ condition.

A Department of Mathematics, University of Oslo, P.O. Box 1053, Blindern, N-0316 Oslo, Norway.

E-mail: kennethk@math.uio.no.

${ }^{B}$ MiraCosta College, 3333 Manchester Avenue, Cardiff-by-the-Sea, CA 92007-1516, USA.

E-mail:john.towers@cox.net. 

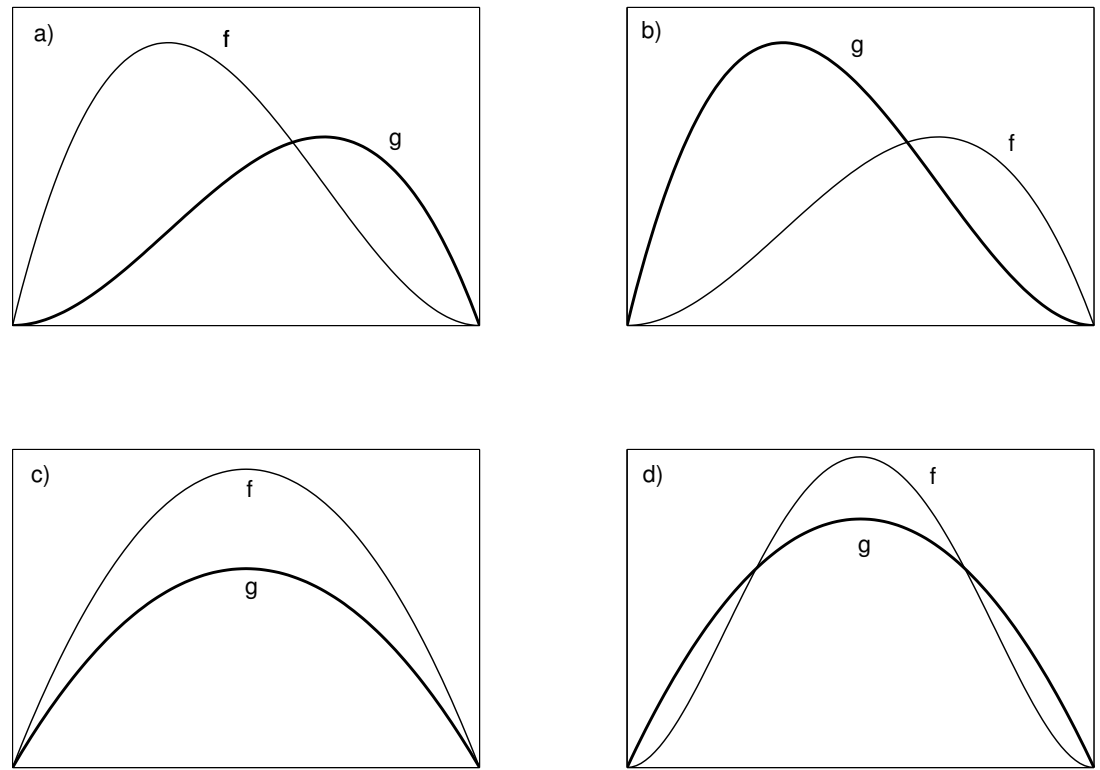

Figure 1. Some simple possibilities for the fluxes $f$ and $g$. There is a single flux crossing in a) and b), no flux crossing in c), and two flux crossings in d). Only b) and c) satisfy the crossing condition.

Definition 1.1 (flux crossing). We call $u_{\chi} \in(\underline{u}, \bar{u})$ a flux crossing if $f\left(u_{\chi}\right)=g\left(u_{\chi}\right)$, and for some $\epsilon>0$ it holds that

$$
\left(g\left(u_{L}\right)-f\left(u_{L}\right)\right)\left(g\left(u_{R}\right)-f\left(u_{R}\right)\right)<0 \text { for all } u_{L} \in\left(u_{\chi}-\epsilon, u_{\chi}\right), u_{R} \in\left(u_{\chi}, u_{\chi}+\epsilon\right) .
$$

Less formally, $u_{\chi}$ is the location of a zero of the function $f(u)-g(u)$ where the sign changes. Throughout this paper, we will make the simplifying assumption that

there are finitely many (possibly zero) flux crossings in the interval $(\underline{u}, \bar{u})$.

Definition 1.2 (Crossing condition). Assume that the functions $f$ and $g$ satisfy all assumptions stated so far. We then say that they satisfy the crossing condition if

$$
\forall u, v \in[\underline{u}, \bar{u}]: \quad f(u)-g(u)<0<f(v)-g(v) \Longrightarrow u<v .
$$

Geometrically, the crossing condition requires that either the graphs of $g$ and $f$ do not cross, or if they do, there is at most one flux crossing $u_{\chi}$, and the graph of $g$ lies above the graph of $f$ to the left of $u_{\chi}$. See Figure 1 .

Even if $f=g$, and even if the initial data is smooth, solutions develop discontinuities, and so weak solutions are sought. Weak solutions are not unique without an additional entropy condition. In the $f=g$ setting, the Kružkov entropy solution is usually sought, and this settles the uniqueness question. Reference 38 proposed a notion of entropy solution for 11.1 . In the context of the present situation, it requires that the following Kružkov-type entropy inequality holds for all $c \in[\underline{u}, \bar{u}]$ and all test functions $0 \leq \phi \in \mathcal{D}\left(\Pi_{T}\right)$ :

$$
\begin{gathered}
\iint_{\Pi_{T}}\left(|u-c| \phi_{t}+\operatorname{sign}(u-c)(\mathcal{F}(x, u)-\mathcal{F}(x, c)) \phi_{x}\right) d x d t \\
\quad+\int_{0}^{T}|f(c)-g(c)| \psi(0, t) d t \geq 0 .
\end{gathered}
$$


It was proven in 38 that the notion of entropy solution defined by 1.8 is sufficient for uniqueness if the crossing condition (1.7) is satisfied, but that if the crossing condition is not satisfied, (1.8) is not restrictive enough. There is an important industrial application where the entropy concept of [38] is well suited, i.e., there is a flux crossing and the crossing condition is satisfied. That is the modeling of clarifier-thickener units [15, 16, 17, 26, 27, 28, 29. Moreover, the entropy definition of 38 has the following useful property: certain finite difference schemes obey a discrete version of 1.8. Using this discrete entropy inequality, it is possible to prove that limits of numerical approximations generated by such a scheme satisfy (1.8), assuming only that they converge boundedly a.e. as the mesh size shrinks to zero. This program is carried out in reference [38, where it is proven that the limit of approximations generated by a very simple class of difference schemes converge to the entropy solution (assuming the crossing condition is satisfied).

Adimurthi, Mishra, and Veerappa Gowda [2] noticed that for the problem (1.1), there is no single notion of entropy solution. Instead, there are infinitely many $L^{1}$-contractive semigroups of solutions. The appropriate notion of entropy solution then becomes a question of modeling. In the case where $f$ and $g$ each have a single extremum, and there is at most one flux crossing, (sometimes referred to as the bell-shaped flux problem, see plots a), b), c) of Figure 1 each type of entropy solution can be characterized by a so-called $A-B$ connection. In this bell-shaped flux setting, a number of papers have proposed finite difference schemes that use a so-called numerical interface flux [2, 4, 15, 8, 10, 19, 41]. The interface flux is designed so that the correct $A-B$ entropy solution is approximated by the numerical scheme. For the bell-shaped problem, the vanishing viscosity solution corresponds to a particular $A-B$ entropy solution, and so it is possible to design an interface flux which yields the vanishing viscosity solution.

There has been ample interest in vanishing viscosity solutions in more complicated situations where the crossing condition is not necessarily satisfied [11, 26, 27, 28, 29, and the fluxes may not be bell-shaped. The present paper is a contribution to that effort. Specifically, we propose the Godunov finite difference scheme of [26, 38, for approximating vanishing viscosity solutions in these more complicated situations. This scheme is very simple since it does not employ an interface flux. From a practical point of view, this means that one can quickly construct an effective numerical scheme by more or less glueing together Godunov schemes for the simpler problems $u_{t}+g(u)_{x}=0$ and $u_{t}+f(u)_{x}=0$. This approach does not require any insight into the solution of the discontinuous flux Riemann problem, which may be complicated if the situation is more complex than the bell-shaped case.

Reference 3] extends the $A-B$ connection approach to the situation where $f$ and $g$ have multiple extrema, and possibly multiple flux crossings. However, in this more general situation, it may be complicated to carry out the $A-B$ connection program in such a way that the resulting interface flux gives the vanishing viscosity solution. For example, we are not aware of any results that provide a correspondence between vanishing viscosity solutions and $A-B$ entropy solutions outside of the bell-shaped setting.

As mentioned above, among the many solution concepts, this paper focuses on the so-called vanishing viscosity solution 6, 7, 10, 11, which results by considering the approximation

$$
u_{t}^{\delta, \epsilon}+\mathcal{F}^{\delta}\left(x, u^{\delta, \epsilon}\right)_{x}=\epsilon u_{x x}^{\delta, \epsilon}, \quad u^{\delta, \epsilon}(x, 0)=u_{0}(x), \quad \delta \downarrow 0, \epsilon \downarrow 0 .
$$

Here $\mathcal{F}^{\delta}$ denotes a suitable regularization of the spatial discontinuity in $\mathcal{F}[6,19,26,27,28$. 20. The vanishing viscosity solution has other characterizations, such as the minimal jump condition of Gimse and Risebro [31, 32], and the $\Gamma$-condition of Diehl [26, 27, 28, 29]. As proved by Andreianov et al. [6], when the crossing condition is satisfied, the entropy solution of [38] is also the vanishing viscosity solution. In other words, the vanishing viscosity solution concept generalizes the solution concept of 38]. Similarly, reference 29] shows that entropy solutions of the type described in [38] satisfy the $\Gamma$ condition whenever the crossing condition holds, so the $\Gamma$ solution concept is more general than the solution concept of [38]. Finally, according to 6 , the $\Gamma$ solution concept is equivalent to the vanishing viscosity concept. The authors of [6] also provide a unifying perspective on many of the entropy conditions in the literature, recovering earlier uniqueness results under weaker conditions as well as new results for other less studied problems. 
Recently a few papers have had as a goal (explicit or implicit) the removal of the crossing condition, see for example [11, 42. In [11] the authors propose a strengthening of $(1.8)$ by incorporating a more involved description of the flux jumps in the entropy inequality, i.e., they suitably modify the last term in (1.8). The resulting entropy condition characterizes the limit of vanishing viscosity approximations, and the authors provide existence and uniqueness results.

In the present paper we utilize a generalization of 1.8 that does the following two things: First, it guarantees uniqueness by singling out the vanishing viscosity solution (see Definition 3.2 below), whether or not the crossing condition is satisfied. Second, it has a discrete version that allows us to prove convergence of the Godunov difference scheme of [26, 38, to the vanishing viscosity solution (independent of whether or not the crossing condition holds). It was claimed by Diehl [26] many years ago that the scheme studied here generates vanishing viscosity solutions. Our results provide a rigorous justification of this claim.

The remaining part of the paper is organized as follows: In Section 2, we introduce and discuss entropy solutions of type $\mathcal{V}$. We show that these entropy solutions coincide with vanishing viscosity solutions in Section 3. In Section 4, we present the Godunov difference scheme. A proof that the scheme converges to the unique vanishing viscosity solution is given in Section 5 . In Section 6 . we call attention to the behavior of characteristic curves at the interface for vanishing viscosity solutions. Several numerical examples are supplied in Section 7

\section{ENTROPY SOLUTIONS OF TYPE $\mathcal{V}$}

As alluded to in the introduction, to account for flux crossings, we will modify the entropy condition 1.8 from 38 . The result is an entropy condition that naturally lends itself to a discrete analog satisfied by certain finite difference schemes, thereby opening up for simple convergence proofs. Influenced by [6, 12, 14, 19, 22, the idea behind our entropy condition is to alter $(1.8)$ by enlarging the usual set of convex entropy functions of the form

$$
\eta(u, c)=|u-c|, \quad c=\text { constant }
$$

to the set

$$
\eta(u, \alpha)=|u-\alpha(x)|, \quad \alpha \in \mathcal{C},
$$

where $\mathcal{C}$ is a certain set of piecewise constant functions that we now define.

Definition 2.1 (the set $\mathcal{C}$ ). The set $\mathcal{C}$ consists of functions $\alpha: \mathbb{R} \rightarrow[\underline{u}, \bar{u}]$ that are either constant, or piecewise constant with exactly one jump, located at $x=0$. If $\alpha(x)=H(-x) c_{-}+H(x) c_{+}$does have a jump $\left(c_{-} \neq c_{+}\right)$, we impose the following restriction on the pair $\left(c_{-}, c_{+}\right)$: There must be at least one flux crossing $u_{\chi}$ between $c_{-}$and $c_{+}$, and then we require that

$$
g\left(c_{-}\right)=\bar{g}\left(u_{\chi}, c_{-}\right), \quad f\left(c_{+}\right)=\bar{f}\left(c_{+}, u_{\chi}\right) .
$$

Given a function $q(u)$, we denote by $\bar{q}(v, u)$ the function

$$
\bar{q}(v, u)= \begin{cases}\min _{w \in[u, v]} q(w), & \text { if } u \leq v \\ \max _{w \in[v, u]} q(w), & \text { if } u>v .\end{cases}
$$

Our notion of entropy solution takes the following form:

Definition 2.2 (Entropy solution of type $\mathcal{V}$ ). A measurable function $u: \Pi_{T} \rightarrow \mathbb{R}$ is an entropy solution of type $\mathcal{V}$ of the initial value problem (1.1) if it satisfies the following conditions:

(D.1) $u \in L^{\infty}\left(\Pi_{T}\right) ; u(x, t) \in[\underline{u}, \bar{u}]$ for a.e. $(x, t) \in \Pi_{T}$.

(D.2) For all test functions $\phi \in \mathcal{D}(\mathbb{R} \times[0, T))$,

$$
\iint_{\Pi_{T}}\left(u \phi_{t}+\mathcal{F}(x, u) \phi_{x}\right) d x d t+\int_{\mathbb{R}} u_{0}(x) \phi(x, 0) d x=0 .
$$


(D.3) For any test function $0 \leq \phi \in \mathcal{D}(\mathbb{R} \times[0, T))$ which vanishes for $x \geq 0$,

$$
\begin{gathered}
\iint_{\Pi_{T}}\left(|u-c| \phi_{t}+\operatorname{sign}(u-c)(g(u)-g(c)) \phi_{x}\right) d x d t \\
+\int_{\mathbb{R}}\left|u_{0}-c\right| \phi(x, 0) d x \geq 0, \quad \forall c \in[\underline{u}, \bar{u}],
\end{gathered}
$$

and for any test function $0 \leq \phi \in \mathcal{D}(\mathbb{R} \times[0, T))$ which vanishes for $x \leq 0$,

$$
\begin{gathered}
\iint_{\Pi_{T}}\left(|u-c| \phi_{t}+\operatorname{sign}(u-c)(f(u)-f(c)) \phi_{x}\right) d x d t \\
+\int_{\mathbb{R}}\left|u_{0}-c\right| \phi(x, 0) d x \geq 0, \quad \forall c \in[\underline{u}, \bar{u}] .
\end{gathered}
$$

(D.4) The following Kružkov-type entropy inequality holds for every $\alpha \in \mathcal{C}$, and for any test function $0 \leq \phi \in \mathcal{D}\left(\Pi_{T}\right)$ :

$$
\begin{gathered}
\iint_{\Pi_{T}}\left(|u-\alpha(x)| \phi_{t}+\operatorname{sign}(u-\alpha(x))(\mathcal{F}(x, u)-\mathcal{F}(x, \alpha(x))) \phi_{x}\right) d x d t \\
+\int_{0}^{T}|f(\alpha(0+))-g(\alpha(0-))| \phi(0, t) d t \geq 0 .
\end{gathered}
$$

A measurable function $u: \Pi_{T} \rightarrow \mathbb{R}$ satisfying conditions (D.1) and (D.2) is called a weak solution of the initial value problem $(1.1)$.

Remark 2.1. Inequality 2.6 is the generalization of 1.8 hinted at previously. Since $\mathcal{C}$ contains constant functions, entropy solutions of type $\mathcal{V}$ satisfy the Kružkov type entropy inequality (1.8) for all $c \in[\underline{u}, \bar{u}]$, meaning that they are entropy solutions in the sense of 38 . With the addition of functions $\alpha(x)$ that are not constant, this solution concept becomes more restrictive than the solution concept of 38, the purpose being to guarantee uniqueness when the crossing condition is not satisfied. By way of comparison, 19 contains an entropy inequality like (2.6). The entropies take the form $\eta(u, \alpha)=|u-\alpha|$ with $\alpha(x)=$ constant, along with the following discontinuous $\alpha(x)$ :

$$
\alpha(x)=c^{A B}(x):=H(-x) A+H(x) B,
$$

where the pair of left and right states $(A, B)$ define the so-called $A-B$ connection. With the simpler setup considered in [19] ( $f$ and $g$ are unimodal with at most one flux crossing), this single discontinuous $\alpha(x)$ (along with the usual constant versions of $\alpha(x)$ ) is sufficient to single out the correct $A-B$ entropy solution sought in that paper. Note that with the setup of [19] only one among the infinitely many $A-B$ connections corresponds to the vanishing viscosity solution. An entropy inequality like (2.6) is also found in Andreianov et al. 6, 7, where it is stated in terms of an "abstract" collection of functions $\alpha(x)$ (germs), rather than the specific set (2.1). The vanishing viscosity solution is examined in [7, without explicitly using this particular entropy inequality.

Remark 2.2. When $\alpha(x)$ has a discontinuity, the function $\eta(u, \alpha)$ is a so-called adapted entropy. The adapted entropy concept has become a standard analytical tool for dealing with discontinuous flux problems like (1.1) 6, 12, 14, 19, 22].

Remark 2.3. We are ultimately interested in vanishing viscosity solutions (cf. Section 3). So let us explain why we are introducing yet another solution concept, i.e., Definition 2.2, as opposed to working directly with the definition of vanishing viscosity solution (see Definition 3.2 below). The definition of vanishing viscosity solution is stated in terms of traces along the interface. For the approximations generated by the Godunov scheme of Section 4 , we have convergence in $L_{\text {loc }}^{1}$ and boundedly a.e., but this mode of convergence does not give convergence of traces, making it difficult to apply the vanishing viscosity definition directly. On the other hand, the Kružkov entropy inequality 2.6 has a discrete analog that is satisfied by the finite difference scheme, and this discrete analog converges to (2.6), assuming only boundedly a.e. convergence. 


\section{ENTROPY SOLUTIONS OF TYPE $\mathcal{V}$ ARE VANISHING VISCOSITY SOLUTIONS}

Let $u$ be an entropy solution of type $\mathcal{V}($ cf. Definition 2.2). We need to discuss certain jump conditions that relate limits from the right and left of $u(\cdot, t)$ at the interface $x=0$. Some type of one-sided limits are required in order for these conditions to make sense. This brings us to the following lemma. We omit the proof since this is basically Lemma 3.1 of [19]. The key ingredient is the genuine nonlinearity assumption 1.5.

Lemma 3.1. Let $u$ be an entropy solution of type $\mathcal{V}$ of $(1.1)$. For a.e. $t \in(0, T)$, the function $u(\cdot, t)$ has strong traces from the left and right at $x=0$, i.e., the following limits exist for a.e. $t \in(0, T)$ :

$$
u\left(0^{-}, t\right):=\underset{x \uparrow 0}{\operatorname{ess} \lim } u(x, t), \quad u\left(0^{+}, t\right):=\underset{x \downarrow 0}{\operatorname{ess}} \lim u(x, t) .
$$

Similarly, $u$ has a strong trace at the initial hyperplane $t=0$.

In the sequel we will often use the abbreviations $u\left(0^{-}, t\right)=u_{-}, u\left(0^{+}, t\right)=u_{+}$for the traces appearing in (3.1). Let $\operatorname{co}(a, b)$ denote the interval $[\min (a, b), \max (a, b)]$. The following is Diehl's $\Gamma$ condition [26, 27, 28, 29].

Definition 3.1 ( $\Gamma$ condition). The pair $\left(u_{-}, u_{+}\right)$satisfies the $\Gamma$ condition if

$$
\begin{aligned}
& g\left(u_{-}\right)=f\left(u_{+}\right) \text {and there exists } \hat{u} \in \operatorname{co}\left(u_{-}, u_{+}\right) \text {such that } \\
& \left(u_{+}-\hat{u}\right)\left(f(z)-f\left(u_{+}\right)\right) \geq 0 \forall z \in \operatorname{co}\left(u_{+}, \hat{u}\right) \text { and } \\
& \left(\hat{u}-u_{-}\right)\left(g(z)-g\left(u_{-}\right)\right) \geq 0 \forall z \in \operatorname{co}\left(u_{-}, \hat{u}\right) .
\end{aligned}
$$

Remark 3.1. The condition $g\left(u_{-}\right)=f\left(u_{+}\right)$appearing in the definition is the familiar RankineHugoniot condition.

The following definition of vanishing viscosity solution is from [7].

Definition 3.2 (vanishing viscosity solution). A function $u: \Pi_{T} \rightarrow \mathbb{R}$ is called a vanishing viscosity solution of (1.1) if it satisfies (D.1), (D.2), (D.3) of Definition 2.2, and in addition, for a.e. $t \in(0, T)$, the traces $u_{-}:=u\left(0^{-}, t\right)$ and $u_{+}:=u\left(0^{+}, t\right)$ satisfy the $\Gamma$ condition.

The vanishing viscosity solution is $L^{1}$ stable and thus unique.

Theorem 3.1 ([6, 7]). Let $u, v$ be two vanishing viscosity solutions in the sense of Definition 3.2 of the initial value problem $\left[1.1\right.$, with initial data $u_{0}, v_{0} \in L^{\infty}(\mathbb{R} ;[0,1]),\left|u_{0}-v_{0}\right| \in L^{1}(\mathbb{R})$. Then, for a.e. $t \in(0, T)$,

$$
\int_{\mathbb{R}}|u(x, t)-v(x, t)| d x \leq \int_{\mathbb{R}}\left|u_{0}(x)-v_{0}(x)\right| d x .
$$

In particular, there exists at most one vanishing viscosity solution of (1.1).

We will show that the $\Gamma$ condition is equivalent to the following condition, which turns out to be more convenient for our analysis. We will use the notation

$$
a \wedge b=\min (a, b), \quad a \vee b=\max (a, b) .
$$

Definition 3.3 ( $\Gamma_{1}$ condition). The pair $\left(u_{-}, u_{+}\right)$satisfies the $\Gamma_{1}$ condition if $g\left(u_{-}\right)=f\left(u_{+}\right)$and

Condition A1. If $u_{-}<u_{+}$, then

$$
g\left(u_{-}\right), f\left(u_{+}\right) \leq g(z) \vee f(z) \text { for all } z \in\left[u_{-}, u_{+}\right] .
$$

Condition A2. If $u_{-}<u_{+}$, and there is a crossing $u_{\chi} \in\left(u_{-}, u_{+}\right)$, then

either $g\left(u_{-}\right), f\left(u_{+}\right) \leq g(z)$ for all $z \in\left[u_{-}, u_{\chi}\right]$,

$$
\text { or } g\left(u_{-}\right), f\left(u_{+}\right) \leq f(z) \text { for all } z \in\left[u_{\chi}, u_{+}\right] \text {. }
$$

Condition B1. If $u_{+}<u_{-}$, then

$$
g\left(u_{-}\right), f\left(u_{+}\right) \geq g(z) \wedge f(z) \text { for all } z \in\left[u_{+}, u_{-}\right] .
$$

Condition B2. If $u_{+}<u_{-}$, and there is a crossing $u_{\chi} \in\left(u_{+}, u_{-}\right)$, then

either $g\left(u_{-}\right), f\left(u_{+}\right) \geq f(z)$ for all $z \in\left[u_{+}, u_{\chi}\right]$,

$$
\text { or } g\left(u_{-}\right), f\left(u_{+}\right) \geq g(z) \text { for all } z \in\left[u_{\chi}, u_{-}\right] \text {. }
$$




\begin{tabular}{l|ll} 
& $g(c) \leq f(c)$ & $g(c) \geq f(c)$ \\
\hline$u_{-} \leq c \leq u_{+}$ & $f\left(u_{+}\right) \leq f(c)$ & $g\left(u_{-}\right) \leq g(c)$ \\
$u_{+} \leq c \leq u_{-}$ & $g\left(u_{-}\right) \geq g(c)$ & $f\left(u_{+}\right) \geq f(c)$
\end{tabular}

TABLE 1. Entropy jump conditions that follow from $\alpha(x)=$ constant $=c \in[\underline{u}, \bar{u}]$. This is basically Table 2 of 38 .

Remark 3.2. Note that if $u_{-}=u_{+}$, both the $\Gamma$ condition and the $\Gamma_{1}$ condition reduce to the Rankine-Hugoniot condition, $g\left(u_{-}\right)=f\left(u_{+}\right)$, which holds at any flux crossing $u_{\chi}$. In other words the constant solution $u(x)=u_{\chi}$ satisfies both the $\Gamma$ condition and the $\Gamma_{1}$ condition. It may happen that $g^{\prime}\left(u_{-}\right)<0<f^{\prime}\left(u_{+}\right)$, i.e., the solution may be very undercompressive at the interface in this case $\left(u_{-}=u_{+}=u_{\chi}\right)$. This type of solution (very undercompressive, continuous across the interface) is admissible for vanishing viscosity solutions, but some other solution concepts do not allow this [1, 2].

We will show that entropy solutions of type $\mathcal{V}$ satisfy the $\Gamma_{1}$ condition. Next, we show that the $\Gamma_{1}$ condition is equivalent to the $\Gamma$ condition. Thus entropy solutions of type $\mathcal{V}$ satisfy the $\Gamma$ condition and (by uniqueness) are therefore vanishing viscosity solutions.

Lemma 3.2. Let $u$ be an entropy solution of type $\mathcal{V}$. Let $t \in(0, T)$ be a time where the traces $u_{-}$ and $u_{+}$exist. Then the Rankine-Hugoniot condition $g\left(u_{-}\right)=f\left(u_{+}\right)$is satisfied, and we have the following entropy jump condition for every $\alpha \in \mathcal{C}$ :

$$
\operatorname{sign}\left(u_{+}-c_{+}\right)\left(f\left(u_{+}\right)-f\left(c_{+}\right)\right)-\operatorname{sign}\left(u_{-}-c_{-}\right)\left(g\left(u_{-}\right)-g\left(c_{-}\right)\right) \leq\left|f\left(c_{+}\right)-g\left(c_{-}\right)\right| .
$$

Proof. The Rankine-Hugoniot condition, $g\left(u_{-}\right)=f\left(u_{+}\right)$follows from (1.8), or (2.3). The proof is a special case of the proof of Lemma 2.4 of [38. The entropy inequality $(3.2)$ is a consequence of (2.6); its proof is similar to the proof of Lemma 2.6 of [38].

Lemma 3.3. Let $u$ be an entropy solution of type $\mathcal{V}$. Let $t \in(0, T)$ be a time where the traces $u_{-}$ and $u_{+}$exist. Then the pair $\left(u_{-}, u_{+}\right)$satisfies the $\Gamma_{1}$ condition.

Proof. The Rankine-Hugoniot condition holds, according to Lemma 3.2 If $u_{-}=u_{+}$, then the $\Gamma_{1}$ condition reduces to the Rankine-Hugoniot condition, so assume that $u_{-} \neq u_{+}$. Taking $\alpha(x)=$ $c \in \operatorname{co}\left(u_{-}, u_{+}\right)$in $(3.2)$, we get Table 1 , as a special case of Lemma 2.7 of 38. Conditions A1 and B1 of the $\Gamma_{1}$ condition are just a reformulation of Table 1.

It remains to verify that Conditions A2 and B2 are satisfied. We first establish a preliminary fact. To this end, let $u_{\chi}$ be a flux crossing, and let $\alpha(x)=H(-x) c_{-}+H(x) c_{+} \in \mathcal{C}$. Assume that $c_{-}, c_{+}, u_{\chi}$ are related by 2.1. We claim that

$$
\text { If } u_{-} \leq c_{-} \leq u_{\chi} \leq c_{+} \leq u_{+} \text {, }
$$

$$
\text { then either } g\left(u_{-}\right) \leq g\left(c_{-}\right) \text {or } f\left(u_{+}\right) \leq f\left(c_{+}\right) \text {. }
$$

$$
\text { If } u_{+} \leq c_{+} \leq u_{\chi} \leq c_{-} \leq u_{-} \text {, }
$$

$$
\text { then either } f\left(u_{+}\right) \geq f\left(c_{+}\right) \text {or } g\left(u_{-}\right) \geq g\left(c_{-}\right) \text {. }
$$

We will prove (3.3). The proof of (3.4) is very similar and we omit it. If $u_{-}=c_{-}$or $u_{+}=c_{+}$, the conclusion of (3.3) holds trivially, so assume

$$
u_{-}<c_{-} \leq u_{\chi} \leq c_{+}<u_{+} .
$$

With this assumption the entropy inequality 3.2 becomes

$$
f\left(u_{+}\right)-f\left(c_{+}\right)+g\left(u_{-}\right)-g\left(c_{-}\right) \leq\left|f\left(c_{+}\right)-g\left(c_{-}\right)\right| .
$$

If $f\left(c_{+}\right) \geq g\left(c_{-}\right)$, with the help of the Rankine-Hugoniot condition, 3.5) simplifies to the relation $f\left(u_{+}\right) \leq f\left(c_{+}\right)$. Similarly, in the case where $f\left(c_{+}\right) \leq g\left(c_{-}\right)$, 3.5 simplifies to $g\left(u_{-}\right) \leq g\left(c_{-}\right)$. Combining these two cases, we have the conclusion of (3.3). 
We now prove that Condition A2 holds if $u_{-}<u_{+}$and there is a crossing $u_{\chi} \in\left(u_{-}, u_{+}\right)$. Define

$$
c_{-} \in \underset{z \in\left[u_{-}, u_{\chi}\right]}{\arg \min } g(z), \quad c_{+} \in \underset{z \in\left[u_{\chi}, u_{+}\right]}{\arg \min } f(z) .
$$

From (3.6) it follows that $\alpha(x):=H(-x) c_{-}+H(x) c_{+} \in \mathcal{C}$, that $c_{-}, c_{+}, u_{\chi}$ are related by 2.1), and that $u_{-} \leq c_{-} \leq u_{\chi} \leq c_{+} \leq u_{+}$. By (3.3), either $g\left(u_{-}\right) \leq g\left(c_{-}\right)$or $f\left(u_{+}\right) \leq f\left(c_{+}\right)$. If $g\left(u_{-}\right) \leq g\left(c_{-}\right)$, then $(3.6)$ implies that $g\left(u_{-}\right) \leq g(z)$ for all $z \in\left[u_{-}, u_{\chi}\right]$. On the other hand, if $f\left(u_{+}\right) \leq f\left(c_{+}\right)$, then (3.6) implies that $f\left(u_{+}\right) \leq f(z)$ for all $z \in\left[u_{\chi}, u_{+}\right]$. Recalling that $g\left(u_{-}\right)=f\left(u_{+}\right)$we see that $\mathrm{A} 2$ holds.

It remains to show that Condition B2 holds if $u_{+}<u_{-}$and there is a crossing $u_{\chi} \in\left(u_{+}, u_{-}\right)$. The proof is similar to the proof of Condition A2 in the preceding paragraph, and we omit it other than to note that instead of $(3.6)$ we define

$$
c_{-} \in \underset{z \in\left[u_{\chi}, u_{-}\right]}{\arg \max } g(z), \quad c_{+} \in \underset{z \in\left[u_{+}, u_{\chi}\right]}{\arg \max } f(z) .
$$

Remark 3.3. Conditions A1 and B1 follow from the Kružkov inequality (1.8). If the crossing condition is satisfied, A1 and B1 are sufficient for uniqueness [38].

We now introduce a change of variables whose purpose is to simplify the proof of Lemma 3.5 Recall that the state space for our problem is $\mathcal{U}=[\underline{u}, \bar{u}]$. The change of variables is:

$$
\begin{aligned}
& \sigma(u):=\bar{u}+\underline{u}-u, \\
& \tilde{g}(z):=-g(\sigma(z))=-g(\bar{u}+\underline{u}-z), \\
& \tilde{f}(z):=-f(\sigma(z))=-f(\bar{u}+\underline{u}-z) .
\end{aligned}
$$

Note that $\sigma(\underline{u})=\bar{u}, \sigma(\bar{u})=\underline{u}$. The function $\sigma$ maps the interval $[\underline{u}, \bar{u}]$ onto itself in a 1-1 manner, $\sigma^{-1}=\sigma$, and we have the following order reversal:

$$
\underline{u} \leq v \leq w \leq \bar{u} \Longleftrightarrow \underline{u}=\sigma(\bar{u}) \leq \sigma(w) \leq \sigma(v) \leq \sigma(\underline{u})=\bar{u},
$$

from which we derive

$$
z \in \operatorname{co}(v, w) \Longleftrightarrow \sigma(z) \in \operatorname{co}(\sigma(v), \sigma(w)), \quad z \in \operatorname{co}(v, w) \Longleftrightarrow \sigma^{-1}(z) \in \operatorname{co}\left(\sigma^{-1}(v), \sigma^{-1}(w)\right) .
$$

We also have the following easily verified relationships:

$$
\begin{aligned}
& \tilde{q}(\sigma(u))=-q(u), \\
& (\sigma(v)-\sigma(u))(\tilde{q}(\sigma(v))-\tilde{q}(\sigma(u)))=(v-u)(q(v)-q(u)),
\end{aligned}
$$

where $q=f, g$. It is readily checked that the Rankine Hugoniot condition is preserved, i.e., $g\left(u_{-}\right)=f\left(u_{+}\right)$iff $\tilde{g}\left(\sigma\left(u_{-}\right)\right)=\tilde{f}\left(\sigma\left(u_{+}\right)\right)$. Moreover, the transformation preserves flux crossings: $u_{\chi}$ is a flux crossing for the fluxes $f, g$ iff $\sigma\left(u_{\chi}\right)$ is a flux crossing for $\tilde{f}, \tilde{g}$.

Lemma 3.4. Part 1. The states $u_{-}, u_{+} \in[\underline{u}, \bar{u}]$ satisfy the $\Gamma$ condition with fluxes $f, g$ iff the states $\sigma\left(u_{-}\right), \sigma\left(u_{+}\right)$satisfy the $\Gamma$ condition with fluxes $\tilde{f}, \tilde{g}$.

Part 2. The states $u_{-}, u_{+} \in[\underline{u}, \bar{u}]$ satisfy the $\Gamma_{1}$ condition with fluxes $f, g$ iff the states $\sigma\left(u_{-}\right)$, $\sigma\left(u_{+}\right)$satisfy the $\Gamma_{1}$ condition with fluxes $\tilde{f}, \tilde{g}$.

Proof. Proof of Part 1. Assume that $u_{-}, u_{+} \in[\underline{u}, \bar{u}]$ satisfy the $\Gamma$ condition with fluxes $f, g$. Since $g\left(u_{-}\right)=f\left(u_{+}\right)$, the first equation of 3.9$)$ gives

$$
\tilde{g}\left(\sigma\left(u_{-}\right)\right)=\tilde{f}\left(\sigma\left(u_{+}\right)\right),
$$

i.e., the Rankine-Hugoniot condition is satisfied. Let $\hat{u}$ be the state guaranteed by the $\Gamma$ condition as it applies to $u_{-}, u_{+}$with fluxes $f, g$. We will show that $\sigma(\hat{u})$ is the state guaranteed by the $\Gamma$ condition as it applies to $\sigma\left(u_{-}\right), \sigma\left(u_{+}\right)$with fluxes $\tilde{f}, \tilde{g}$. Take $z \in \operatorname{co}\left(\sigma\left(u_{+}\right), \sigma(\hat{u})\right)$. It follows from (3.8) that $\sigma^{-1}(z) \in \operatorname{co}\left(u_{+}, \hat{u}\right)$. Invoking the $\Gamma$ condition (for $u_{-}, u_{+}$with fluxes $f, g$ ), we have

$$
\left(u_{+}-\hat{u}\right)\left(f\left(\sigma^{-1}(z)\right)-f\left(u_{+}\right)\right) \geq 0 .
$$


Now invoking the second relationship of $(3.9)$, we get

$$
\left(\sigma\left(u_{+}\right)-\sigma(\hat{u})\right)\left(\tilde{f}(z)-\tilde{f}\left(\sigma\left(u_{+}\right)\right)\right) \geq 0, \quad \forall z \in \operatorname{co}\left(\sigma\left(u_{+}\right), \sigma(\hat{u})\right) .
$$

A similar calculation gives

$$
\left(\sigma(\hat{u})-\sigma\left(u_{-}\right)\right)\left(\tilde{g}(z)-\tilde{g}\left(\sigma\left(u_{-}\right)\right)\right) \geq 0, \quad \forall z \in \operatorname{co}\left(\sigma\left(u_{-}\right), \sigma(\hat{u})\right) .
$$

Combining (3.10, 3.11) and (3.12), we have shown that the $\Gamma$ condition holds for $\sigma\left(u_{-}\right), \sigma\left(u_{+}\right)$ with fluxes $f, \tilde{g}$. The proof of the converse is similar, and we omit it.

Proof of Part 2. Now assume that $u_{-}, u_{+} \in[\underline{u}, \bar{u}]$ satisfy the $\Gamma_{1}$ condition with fluxes $f, g$. The Rankine-Hugoniot condition 3.10 holds for the same reason as above. If $u_{-}=u_{+}$, the $\Gamma_{1}$ condition reduces to the Rankine-Hugoniot condition, so assume that $u_{-} \neq u_{+}$. Assume that $u_{-}<u_{+}$; the proof when $u_{+}<u_{-}$is similar and we omit it. Invoking Condition A1 of the $\Gamma_{1}$ condition (applied to $u_{-}, u_{+}$with fluxes $f, g$ ), and then using the first relationship of (3.9), we get

$$
-\tilde{g}\left(\sigma\left(u_{-}\right)\right),-\tilde{f}\left(\sigma\left(u_{+}\right)\right) \leq-\tilde{g}(\sigma(z)) \vee-\tilde{f}(\sigma(z)), \quad \forall z \in\left[u_{-}, u_{+}\right] .
$$

We multiply by -1 , and use the fact that $z \in\left[u_{-}, u_{+}\right]$iff $\sigma(z) \in\left[\sigma\left(u_{+}\right), \sigma\left(u_{-}\right)\right]$, yielding

$$
\tilde{g}\left(\sigma\left(u_{-}\right)\right), \tilde{f}\left(\sigma\left(u_{+}\right)\right) \geq \tilde{g}(\zeta) \wedge \tilde{f}(\zeta), \quad \forall \zeta \in\left[\sigma\left(u_{+}\right), \sigma\left(u_{-}\right)\right] .
$$

Since $\sigma(u)_{+}<\sigma(u)_{-}$iff $u_{-}<u_{+}$, we have shown that $\sigma\left(u_{-}\right), \sigma\left(u_{+}\right)$with fluxes $\tilde{f}, \tilde{g}$ satisfy the $\Gamma_{1}$ condition (specifically Condition B1).

Continuing to assume that $u_{-}<u_{+}$, we will next show that $\sigma\left(u_{-}\right), \sigma\left(u_{+}\right)$with fluxes $\tilde{f}, \tilde{g}$ satisfy Condition B2 of the $\Gamma_{1}$ condition. Assume that there is a crossing $u_{\chi}$ of $\tilde{f}, \tilde{g}$, and $u_{\chi} \in$ $\left(\sigma\left(u_{+}\right), \sigma\left(u_{-}\right)\right)$. Then $\sigma^{-1}\left(u_{\chi}\right) \in\left(u_{-}, u_{+}\right)$, and $\sigma^{-1}\left(u_{\chi}\right)$ is a crossing of $f, g$. Invoking Condition A2 (as applied to $u_{-}, u_{+}$with fluxes $f, g$ ), we have

$$
\begin{gathered}
\text { either } g\left(u_{-}\right), f\left(u_{+}\right) \leq g(z) \text { for all } z \in\left[u_{-}, \sigma^{-1}\left(u_{\chi}\right)\right] \\
\text { or } g\left(u_{-}\right), f\left(u_{+}\right) \leq f(z) \text { for all } z \in\left[\sigma^{-1}\left(u_{\chi}\right), u_{+}\right]
\end{gathered}
$$

Applying $\sigma(\cdot)$, along with the first relationship of 3.9 , we find that

$$
\begin{array}{r}
\text { either } \tilde{g}\left(\sigma\left(u_{-}\right)\right), \tilde{f}\left(\sigma\left(u_{+}\right)\right) \geq \tilde{g}(\sigma(z)) \text { for all } z \in\left[u_{-}, \sigma^{-1}\left(u_{\chi}\right)\right], \\
\quad \text { or } \tilde{g}\left(\sigma\left(u_{-}\right)\right), \tilde{f}\left(\sigma\left(u_{+}\right)\right) \geq \tilde{f}(\sigma(z)) \text { for all } z \in\left[\sigma^{-1}\left(u_{\chi}\right), u_{+}\right]
\end{array}
$$

Recalling that $\sigma(u)_{+}<\sigma(u)_{-}$, and using

$$
z \in\left[u_{-}, \sigma^{-1}\left(u_{\chi}\right)\right] \text { iff } \sigma(z) \in\left[u_{\chi}, \sigma\left(u_{-}\right)\right], \quad z \in\left[\sigma^{-1}\left(u_{\chi}\right), u_{+}\right] \text {iff } \sigma(z) \in\left[\sigma\left(u_{+}\right), u_{\chi}\right],
$$

we find from 3.13 that

$$
\begin{array}{r}
\text { either } \tilde{g}\left(\sigma\left(u_{-}\right)\right), \tilde{f}\left(\sigma\left(u_{+}\right)\right) \geq \tilde{g}(\zeta) \text { for all } \zeta \in\left[u_{\chi}, \sigma\left(u_{-}\right)\right], \\
\quad \text { or } \tilde{g}\left(\sigma\left(u_{-}\right)\right), \tilde{f}\left(\sigma\left(u_{+}\right)\right) \geq \tilde{f}(\zeta) \text { for all } \zeta \in\left[\sigma\left(u_{+}\right), u_{\chi}\right],
\end{array}
$$

which is Condition B2 of the $\Gamma_{1}$ condition for $\sigma\left(u_{-}\right), \sigma\left(u_{+}\right)$with fluxes $\tilde{f}, \tilde{g}$.

The proof of the converse is similar and we omit it.

Lemma 3.5. The $\Gamma$ condition and the $\Gamma_{1}$ condition are equivalent.

Proof. First note that if $u_{-}=u_{+}$, then both the $\Gamma$ condition and the $\Gamma_{1}$ condition are trivially true iff the Rankine-Hugoniot condition $g\left(u_{-}\right)=f\left(u_{+}\right)$is true, so assume that $u_{-} \neq u_{+}$.

Part $1\left(\Gamma_{1} \Longrightarrow \Gamma\right)$. Assume the $\Gamma_{1}$ condition is satisfied for a pair of states $\left(u_{-}, u_{+}\right)$. For now we will focus on the case where $u_{-}<u_{+}$, basing the proof on conditions A1 and A2 of the $\Gamma_{1}$ condition.

If there are no flux crossings in $\left(u_{-}, u_{+}\right)$, then either $g(z) \geq f(z)$ for all $z \in\left[u_{-}, u_{+}\right]$(see plot a) of Figure 2), or $g(z) \leq f(z)$ for all $z \in\left[u_{-}, u_{+}\right]$(see plot b) of Figure 2). In the first case, condition A1 asserts that $g(z) \geq g\left(u_{-}\right)$for all $z \in\left[u_{-}, u_{+}\right]$, and so we can satisfy the $\Gamma$ condition with $\hat{u}=u_{+}$. In the second case, condition A1 asserts that $f(z) \geq f\left(u_{+}\right)$for all $z \in\left[u_{-}, u_{+}\right]$, and so we can take $\hat{u}=u_{-}$. 

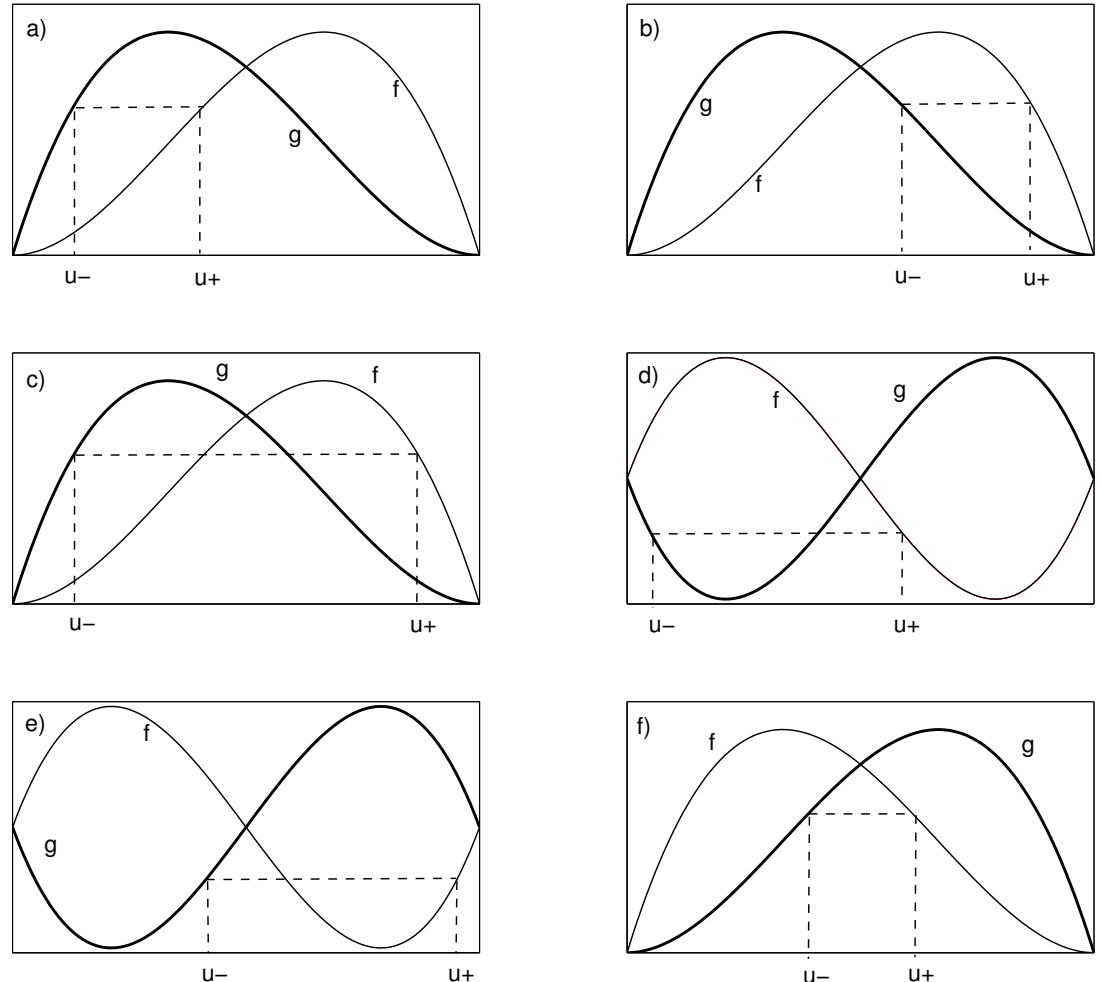

Figure 2. Proof of Lemma 3.5 when $u_{-}<u_{+}$. Plots a), b): no flux crossing in $\left(u_{-}, u_{+}\right)$. Plot c): Single flux crossing in $\left(u_{-}, u_{+}\right)$and $(3.14)$ holds. Plot d), e), f): Single flux crossing in $\left(u_{-}, u_{+}\right)$and $(3.15)$ holds.

Suppose now that there is exactly one flux crossing in $\left(u_{-}, u_{+}\right)$, call it $u_{\chi}$. There are two possibilities. Either

$$
f(z) \leq g(z) \forall z \in\left[u_{-}, u_{\chi}\right], \quad g(z) \leq f(z) \forall z \in\left[u_{\chi}, u_{+}\right],
$$

or

$$
g(z) \leq f(z) \forall z \in\left[u_{-}, u_{\chi}\right], \quad f(z) \leq g(z) \forall z \in\left[u_{\chi}, u_{+}\right] .
$$

If the situation is (3.14), see plot c) of Figure 2 , then by condition A1, we must have $g(z) \geq g\left(u_{-}\right)$ for all $z \in\left[u_{-}, u_{\chi}\right]$, and $f(z) \geq f\left(u_{+}\right)$for all $z \in\left[u_{\chi}, u_{+}\right]$. Thus, we can satisfy the $\Gamma$ condition with $\hat{u}=u_{\chi}$. If $(3.15$ holds (see plots $\mathrm{d}$ ), e), f) of Figure 2), then by condition A1, we must have $f(z) \geq g\left(u_{-}\right)=f\left(u_{+}\right)$for all $z \in\left[u_{-}, u_{\chi}\right]$, and $g(z) \geq f\left(u_{+}\right)=g\left(u_{-}\right)$for all $z \in\left[u_{\chi}, u_{+}\right]$. We now invoke condition A2. Either $f(z) \geq f\left(u_{+}\right)$for all $z \in\left[u_{\chi}, u_{+}\right]$(plots d), f) of Figure 2), or $g(z) \geq g\left(u_{-}\right)$for all $z \in\left[u_{-}, u_{\chi}\right]$ (plots e), f) of Figure 2). In the first situation, A1 and A2 together give $f(z) \geq f\left(u_{+}\right)$for all $z \in\left[u_{-}, u_{+}\right]$, so we take $\hat{u}=u_{-}$. In the second case, A1 and A2 together give $g(z) \geq g\left(u_{-}\right)$for all $z \in\left[u_{-}, u_{+}\right]$, so we take $\hat{u}=u_{+}$.

Still assuming that $u_{-}<u_{+}$, consider the remaining case where there are at least two flux crossings in $\left(u_{-}, u_{+}\right)$. For this part of the proof, we use the notation $X$ for a flux crossing. If $X$ is a crossing, then by condition A2, either $g\left(u_{-}\right) \leq g(z)$ for $z \in\left[u_{-}, X\right]$, or $f\left(u_{+}\right) \leq f(z)$ for $z \in\left[X, u_{+}\right]$. In the first case, we say that $X$ has property $\mathrm{G}$, and in the second case $X$ has property F. Note that a particular crossing may have both properties, and that by condition A2, it must 

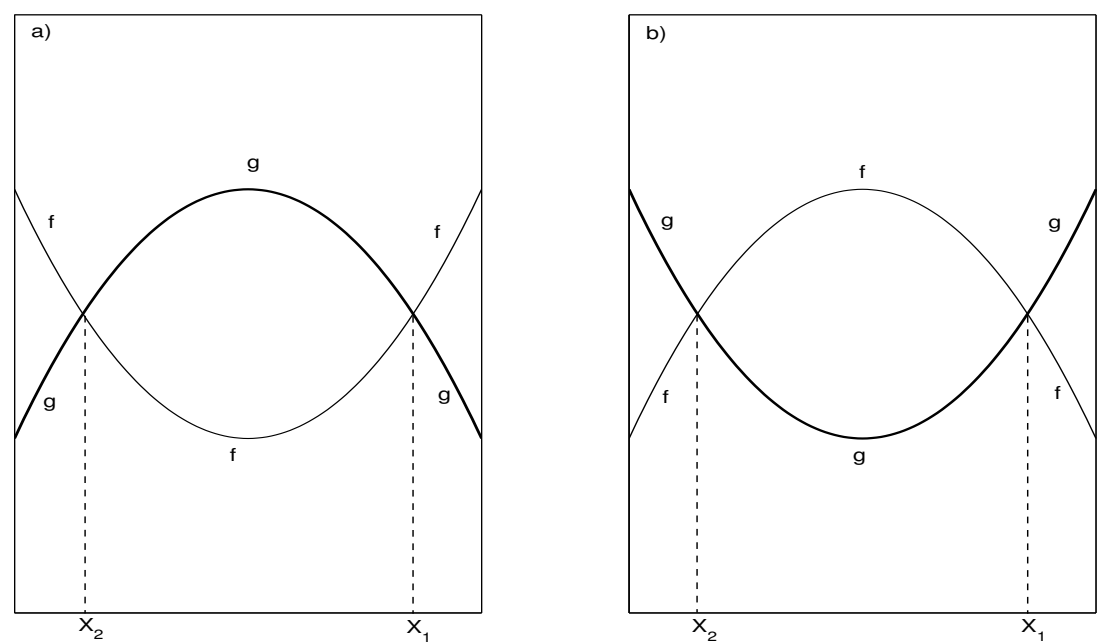

Figure 3. Proof of Lemma 3.5 when $u_{-}<u_{+}$. Plots of $f$ and $g$ near the consecutive flux crossings $X_{1}$ and $X_{2}$. The figures are meant to convey that there are possibly additional flux crossings outside of the interval $\left[X_{2}, X_{1}\right]$, which are not shown. Plot a) shows Configuration 1. Plot b) shows Configuration 2.

have at least one of them. Define the following two subsets of crossings $X$ that lie in $\left(u_{-}, u_{+}\right)$:

$S_{F}=\left\{X \in\left(u_{-}, u_{+}\right): X\right.$ has property $\left.\mathrm{F}\right\}, \quad S_{G}=\left\{X \in\left(u_{-}, u_{+}\right): X\right.$ has property G $\}$.

Note that at least one of $S_{F}, S_{G}$ must be nonempty.

For now assume that $S_{F} \neq \emptyset, S_{G} \neq \emptyset$. Let

$$
X_{1}=\min \left\{X \in S_{F}\right\}, \quad X_{2}=\max \left\{X \in S_{G}\right\} .
$$

Case $1\left(X_{1} \leq X_{2}\right)$. In this case, $f\left(u_{+}\right) \leq f(z)$ for $z \in\left[X_{1}, u_{+}\right], g\left(u_{-}\right) \leq g(z)$ for $z \in\left[u_{-}, X_{2}\right]$. This makes it clear that we can satisfy the $\Gamma$ condition using $\hat{u}=X_{1}$, or $\hat{u}=X_{2}$, or in fact any number in $\left[X_{1}, X_{2}\right]$.

Case $2\left(X_{2}<X_{1}\right)$. We claim that this case is impossible. Assuming otherwise, then $X_{2}, X_{1}$ must be consecutive crossings (no crossings between them). To see this, note that if there were a flux crossing between $X_{2}$ and $X_{1}$, it would have either property G or property F (possibly both), contradicting (3.16). Thus, Case 2 boils down to the following two configurations (See Figure 3):

Configuration 1:

$$
\begin{array}{ccccccc}
\cdots & f & & g & & f & \cdots \\
\cdots & X_{2} & & X_{1} & \cdots & \\
\cdots & g & & f & & g & \cdots
\end{array}
$$

Configuration 2:

$$
\begin{array}{ccccccc}
\cdots & g & & f & & g & \cdots \\
\cdots & X_{2} & & X_{1} & \cdots & \\
\cdots & f & & g & & f & \cdots
\end{array}
$$

By condition A1, in Configuration $1 g(z) \geq g\left(u_{-}\right)$for $z \in\left[X_{2}, X_{1}\right]$, and in Configuration 2, $f(z) \geq f\left(u_{+}\right)$for $z \in\left[X_{2}, X_{1}\right]$. Thus, in the case of Configuration 1 , we see that $X_{1}$ has property $\mathrm{G}$ (due to condition $\mathrm{A} 1$ and the definition of $X_{2}$ ), contradicting the definition of $X_{2}$, and for 
Configuration 2, it is clear that $X_{2}$ has property $\mathrm{F}$ (due to condition A1 and the definition of $X_{1}$ ), contradicting the definition of $X_{1}$.

Now assume that one of $S_{F}, S_{G}$ is empty. We will focus on the case where $S_{F}=\emptyset, S_{G} \neq \emptyset$, the other case being similar. Consider the crossing $X_{2}$ defined by (3.16). Two configurations are seemingly possible:

$$
\begin{aligned}
& \text { Configuration } 1^{*} \text { : } \\
& \begin{array}{ccccc}
\cdots & g & & f & \cdots \\
\cdots & X_{2} & \cdots & \\
\cdots & f & & g & \cdots
\end{array}
\end{aligned}
$$

Configuration $2^{*}$ :

$$
\begin{array}{ccccc}
\cdots & f & & g & \cdots \\
\cdots & X_{2} & \cdots & \\
\cdots & g & & f & \cdots
\end{array}
$$

Note that since $S_{F}=\emptyset$, there are no crossings between $X_{2}$ and $u_{+}$. (If there were such a crossing, it would have to be of Type $\mathrm{G}$, but that would contradict the definition of $X_{2}$.) Thus for Configuration $1^{*}$, Condition A1 (applied to $u_{\chi}=X_{2}$ ) implies that

$$
f\left(u_{+}\right) \leq f(z) \text { for all } z \in\left[X_{2}, u_{+}\right],
$$

and for Configuration $2^{*}$, Condition A1 implies that

$$
g\left(u_{-}\right) \leq g(z) \text { for all } z \in\left[X_{2}, u_{+}\right] .
$$

It turns out that Configuration $1^{*}$ is impossible. To see this, note that due to (3.17), $X_{2}$ satisfies Condition $F$, violating our assumption that $S_{F}=\emptyset$. So assume that we have Configuration $2^{*}$. Since $X_{2}$ satisfies Condition $G$, we have $g\left(u_{-}\right) \leq g(z)$ for $z \in\left[u_{-}, X_{2}\right]$. Combining this with (3.18), we have $g\left(u_{-}\right) \leq g(z)$ for $z \in\left[u_{-}, u_{+}\right]$. So in this case we can satisfy Condition $\Gamma$ with $\hat{u}=u_{+}$. This completes the proof of Part 1 in the case where $u_{-}<u_{+}$.

Now consider the case where $u_{+}<u_{-}$. We use the change of variables (3.7) to deal with this situation. By Lemma 3.4 (Part 2) the $\Gamma_{1}$ condition holds for the states $\sigma\left(u_{-}\right), \sigma\left(u_{+}\right)$with fluxes $\tilde{f}, \tilde{g}$. Observing that $\sigma\left(u_{-}\right)<\sigma\left(u_{+}\right)$, we can apply the previous argument to conclude that the $\Gamma$ condition holds for the states $\sigma\left(u_{-}\right), \sigma\left(u_{+}\right)$with fluxes $\tilde{f}, \tilde{g}$. By Lemma 3.4 (Part 1) the $\Gamma$ condition holds for the states $u_{-}, u_{+}$with fluxes $f, g$.

Part $2\left(\Gamma \Longrightarrow \Gamma_{1}\right)$. Now assume that the $\Gamma$ condition is satisfied for a pair of states $\left(u_{-}, u_{+}\right)$. Again, we first focus on the case where $u_{-}<u_{+}$. The $\Gamma$ condition provides a $\hat{u} \in\left[u_{-}, u_{+}\right]$such that

$$
g(z) \geq g\left(u_{-}\right), \quad \forall z \in\left[u_{-}, \hat{u}\right], \quad f(z) \geq f\left(u_{+}\right), \quad \forall z \in\left[\hat{u}, u_{+}\right] .
$$

These relationships remain true if we substitute $g(z) \vee f(z)$ for $g(z)$ in the first inequality and for $f(z)$ in the second inequality, yielding

$$
g(z) \vee f(z) \geq g\left(u_{-}\right), \quad \forall z \in\left[u_{-}, \hat{u}\right], \quad g(z) \vee f(z) \geq f\left(u_{+}\right), \quad \forall z \in\left[\hat{u}, u_{+}\right] .
$$

Recalling that $g\left(u_{-}\right)=f\left(u_{+}\right)$, we can combine these two inequalities, proving condition A1.

To prove condition A2, let $u_{\chi}$ be a crossing, with $u_{\chi} \in\left(u_{-}, u_{+}\right)$. Again the $\Gamma$ condition guarantees the existence of a $\hat{u} \in\left[u_{-}, u_{+}\right]$satisfying $(3.19)$. If $\hat{u} \leq u_{\chi}$, then the second inequality of (3.19) implies that $f(z) \geq f\left(u_{+}\right) \forall z \in\left[u_{\chi}, u_{+}\right]$, and condition A2 is satisfied. On the other hand, if $\hat{u} \geq u_{\chi}$, then the first inequality of $\left[3.19\right.$ implies that $g(z) \geq g\left(u_{-}\right) \forall z \in\left[u_{-}, u_{\chi}\right]$, and condition A2 is satisfied in this case also.

Now consider the case where $u_{+}<u_{-}$. By Lemma 3.4 (Part 1) the $\Gamma$ condition holds for the states $\sigma\left(u_{-}\right), \sigma\left(u_{+}\right)$with fluxes $\tilde{f}, \tilde{g}$. Observing that $\sigma\left(u_{-}\right)<\sigma\left(u_{+}\right)$, we can apply the previous argument to conclude that the $\Gamma_{1}$ condition holds for the states $\sigma\left(u_{-}\right), \sigma\left(u_{+}\right)$with fluxes $\tilde{f}, \tilde{g}$. By Lemma 3.4 (Part 2) the $\Gamma_{1}$ condition holds for the states $u_{-}, u_{+}$with fluxes $f, g$.

We have the following theorem, which follows directly from Lemmas 3.3 and 3.5 
Theorem 3.2. If $u$ is an entropy solution of type $\mathcal{V}$, then $u$ is a vanishing viscosity solution.

\section{A Godunov scheme}

In this section, we define a simple monotone scheme based on the scalar two-point Godunov numerical flux. We begin by discretizing the spatial domain $\mathbb{R}$ into cells

$$
I_{j}=\left(x_{j}-\Delta x / 2, x_{j}+\Delta x / 2\right]=\left(x_{j-\frac{1}{2}}, x_{j+\frac{1}{2}}\right],
$$

where $x_{j}=j \Delta x$ for $j \in \mathbf{Z}$. Similarly, the time interval $[0, T]$ is is discretized via $t^{n}=n \Delta t$ for $n=0, \ldots, N$, where the integer $N$ is such that $N \Delta t \in[T, t+\Delta t)$, resulting in the time strips

$$
I^{n}=\left[t^{n}, t^{n+1}\right) .
$$

Here $\Delta x>0$ and $\Delta t>0$ denote the spatial and temporal discretization parameters respectively. With $\lambda=\Delta t / \Delta x$, we will assume that the mesh size $\Delta:=(\Delta x, \Delta t)$ approaches zero with $\lambda$ fixed. We will assume that the following CFL condition holds:

$$
\lambda \cdot \max \left(L_{g}, L_{f}\right) \leq 1 .
$$

Let $\chi_{j}(x)$ and $\chi^{n}(t)$ be the characteristic functions for the intervals $I_{j}$ and $I^{n}$, respectively. Define $\chi_{j}^{n}(x, t)=\chi_{j}(x) \chi^{n}(t)$ to be the characteristic function for the rectangle

$$
R_{j}^{n}=I_{j} \times I^{n} .
$$

We will use $U_{j}^{n}$ to denote the finite difference approximation of $u(j \Delta x, n \Delta t)$. The iteration is started by setting

$$
U_{j}^{0}=\frac{1}{\Delta x} \int_{x_{j-\frac{1}{2}}}^{x_{j+\frac{1}{2}}} u_{0}(x) d x .
$$

The finite difference solution $\left\{U_{j}^{n}\right\}$ is extended to all of $\Pi_{T}$ by defining

$$
u^{\Delta}(x, t)=\sum_{n=0}^{N} \sum_{j \in \mathbf{Z}} \chi_{j}^{n}(x, t) U_{j}^{n}, \quad(x, t) \in \Pi_{T},
$$

where $\Delta=(\Delta x, \Delta t)$. We use $\Delta_{+}$and $\Delta_{-}$to designate the difference operators in the $x$ direction, e.g.,

$$
\Delta_{+} Z_{j}=Z_{j+1}-Z_{j}, \quad \Delta_{-} Z_{j}=Z_{j}-Z_{j-1} .
$$

The approximate solution is advanced from one time level to the next via the following finite difference formula

$$
U_{j}^{n+1}=U_{j}^{n}-\lambda \Delta_{-} h\left(x_{j+\frac{1}{2}}, U_{j+1}^{n}, U_{j}^{n}\right),
$$

where $h$ is the spatially dependent Godunov numerical flux:

$$
h\left(x_{j+\frac{1}{2}}, v, u\right)= \begin{cases}\bar{g}(v, u), & \text { if } x_{j+\frac{1}{2}}<0, \\ \bar{f}(v, u), & \text { if } x_{j+\frac{1}{2}}>0 .\end{cases}
$$

Here $\bar{f}, \bar{g}$ refer to the Godunov fluxes consistent with $f, g$ defined by 2.2 .

Remark 4.1. The interface $x=0$, where the flux changes type, falls at the midpoint of the cell $I_{0}=\left(x_{-\frac{1}{2}}, x_{\frac{1}{2}}\right]$. Thus, although complicated waves may arise at the interface, the scheme averages them out. This is the reason that a so-called interface flux is not needed, and it accounts for the relative simplicity of the scheme. This is basically the staggered mesh approach that has already appeared in a number of works concerning discontinuous (and rough) flux problems [17, 20, 21, 26, 35, 37, 38, 46, 47]. The scheme was first proposed by Diehl in [26] as a way of justifying the $\Gamma$ condition. 


\section{CONVERGEnCE TO The VANiShing VisCosity SOLution}

We will show that approximations produced by the scheme of Section 4 converge to an entropy solution of type $\mathcal{V}$, which according to Section 3 , is also a vanishing viscosity solution. For now we assume that the initial data $u_{0} \in \mathrm{BV}(\mathbb{R})$ in addition to 1.2 . Thanks to Theorem 3.1 there is no loss of generality in doing so. For simplicity we also assume that $u_{0}$ is compactly supported, which implies that all subsequent sums are finite. To obtain results in the general case, we can again use Therorem 3.1 .

A finite difference scheme such as the scheme 44.3 ) is monotone [33, 40] if

$$
U_{j}^{n} \leq W_{j}^{n} \quad \forall j \in \mathbb{Z} \quad \Longrightarrow \quad U_{j}^{n+1} \leq W_{j}^{n+1} \quad \forall j \in \mathbb{Z} .
$$

Let $V_{a}^{b}(z)$ denote the total variation of the function $x \mapsto z(x)$ over the interval $(a, b)$. The following lemma provides the main properties required for compactness. We omit the proof, which is similar to the proof of Lemmas 5.1, 5.2, and 5.3 of [19].

Lemma 5.1. The scheme of Section 4 is monotone, and the solution computed by the scheme satisfies the following properties:

$$
U_{j}^{n} \in[\underline{u}, \bar{u}], \quad j \in \mathbb{Z}, n=0,1,2, \ldots
$$

There exists a constant $C_{1}$, depending on $T V\left(u_{0}\right)$, but independent of $\Delta$ and $n$, such that

$$
\Delta x \sum_{\mathrm{j} \in \mathbf{Z}}\left|U_{j}^{n+1}-U_{j}^{n}\right| \leq \Delta x \sum_{\mathrm{j} \in \mathbf{Z}}\left|U_{j}^{1}-U_{j}^{0}\right| \leq C_{1} \Delta t .
$$

For each $a>0$, there is a constant $C_{2}(a)$ such that

$$
V_{-\infty}^{-a}\left(u^{\Delta}(\cdot, t)\right) \leq C_{2}(a), \quad V_{a}^{\infty}\left(u^{\Delta}(\cdot, t)\right) \leq C_{2}(a),
$$

where $C_{2}(a)$ is independent of $\Delta$.

Lemma 5.2. For the scheme of Section 4, we have the following discrete entropy inequality for any $C_{j-1}, C_{j}, C_{j+1} \in[\underline{u}, \bar{u}]$ :

$$
\left|U_{j}^{n+1}-C_{j}\right| \leq\left|U_{j}^{n}-C_{j}\right|-\lambda \Delta_{-} H_{j+\frac{1}{2}}^{n}-\lambda \operatorname{sign}\left(U_{j}^{n+1}-C_{j}\right) \Delta_{-} h\left(x_{j+\frac{1}{2}}, C_{j+1}, C_{j}\right),
$$

where the numerical entropy flux $H_{j+\frac{1}{2}}^{n}$ is defined by

$$
H_{j+\frac{1}{2}}=h\left(x_{j+\frac{1}{2}}, U_{j+1}^{n} \vee C_{j+1}, U_{j}^{n} \vee C_{j}\right)-h\left(x_{j+\frac{1}{2}}, U_{j+1}^{n} \wedge C_{j+1}, U_{j}^{n} \wedge C_{j}\right) .
$$

Proof. Let

$$
G_{j}\left(U_{j+1}^{n}, U_{j}^{n}, U_{j-1}^{n}\right)=U_{j}^{n}-\lambda \Delta_{-} h\left(x_{j+\frac{1}{2}}, U_{j+1}^{n}, U_{j}^{n}\right) .
$$

By Lemma 5.1. $G_{j}$ is monotone in each of its three arguments. Monotonicity implies that

$$
\begin{aligned}
& G_{j}\left(U_{j+1}^{n} \vee C_{j+1}, U_{j}^{n} \vee C_{j}, U_{j-1}^{n} \vee C_{j-1}\right) \geq G_{j}\left(U_{j+1}^{n}, U_{j}^{n}, U_{j-1}^{n}\right) \vee G_{j}\left(C_{j+1}, C_{j}, C_{j-1}\right) \\
& G_{j}\left(U_{j+1}^{n} \wedge C_{j+1}, U_{j}^{n} \wedge C_{j}, U_{j-1}^{n} \wedge C_{j-1}\right) \leq G_{j}\left(U_{j+1}^{n}, U_{j}^{n}, U_{j-1}^{n}\right) \wedge G_{j}\left(C_{j+1}, C_{j}, C_{j-1}\right) .
\end{aligned}
$$

Subtracting (5.4) from (5.3), we obtain

$$
\left|G_{j}\left(U_{j+1}^{n}, U_{j}^{n}, U_{j-1}^{n}\right)-G_{j}\left(C_{j+1}, C_{j}, C_{j-1}\right)\right| \leq\left|U_{j}^{n}-C_{j}\right|-\lambda \Delta_{-} H_{j+\frac{1}{2}} .
$$

The left side of 5.5. simplifies to $\left|U_{j}^{n+1}-C_{j}+\lambda_{-} h\left(x_{j+\frac{1}{2}}, C_{j+1}, C_{j}\right)\right|$, and

$$
\begin{aligned}
& \left|U_{j}^{n+1}-C_{j}+\lambda \Delta_{-} h\left(x_{j+\frac{1}{2}}, C_{j+1}, C_{j}\right)\right| \\
& \quad \geq \operatorname{sign}\left(U_{j}^{n+1}-C\right)\left(U_{j}^{n+1}-C_{j}+\lambda \Delta_{-} h\left(x_{j+\frac{1}{2}}, C_{j+1}, C_{j}\right)\right) \\
& \quad=\left|U_{j}^{n+1}-C_{j}\right|+\lambda \operatorname{sign}\left(U_{j}^{n+1}-C_{j}\right) \Delta_{-} h\left(x_{j+\frac{1}{2}}, C_{j+1}, C_{j}\right) .
\end{aligned}
$$

The proof is completed by combining (5.5) and 5.6).

Lemma 5.3. Suppose that $u^{\Delta}$ generated by the scheme of Section 4 converges boundedly a.e. to $u$, with $u(x, t) \in[\underline{u}, \bar{u}]$ for a.e. $(x, t) \in \Pi_{T}$. Then $u$ is an entropy solution of type $\mathcal{V}$. 
Proof. Condition (D.1) of Definition 2.2 is satisfied by assumption. The proof of Condition (D.2) is a standard Lax-Wendroff type calculation, and we omit it. The proof of Condition (D.3) is also standard 23] by taking $\alpha(x)=c$, and choosing test functions that vanish in $x \geq 0$ (for the integral inequality (2.4), and then in $x \leq 0$ (for (2.5)).

Thus it remains to prove Condition (D.4) Let $0 \leq \phi \in \mathcal{D}\left(\Pi_{T}\right)$ with $\left.\phi\right|_{t=0}=\left.\phi\right|_{t=T}=0$. Fix $X>0$ such that $\phi$ vanishes for $|x| \geq X$. Define the positive integers $J$ and $N$ by requiring that $J \Delta x \in[X, X+\Delta x)$ and $N \Delta t \in[T, T+\Delta t)$. Set $\phi_{j}^{n}=\phi\left(x_{j}, t^{n}\right)$. Let $\alpha \in \mathcal{C}$. If $\alpha(x)=c$, i.e., $\alpha$ is constant, we discretize it in the obvious way: $C_{j}=c, j \in \mathbb{Z}$. When $\alpha$ has a jump, $\alpha(x)=H(-x) c_{-}+H(x) c_{+}$, and according to Definition 2.1 there is a flux crossing $u_{\chi}$ lying between $c_{-}$and $c_{+}$. In addition $c_{-}, c_{+}, u_{\chi}$ are related by (2.1). In this case, the discretization that we use is

$$
C_{j}= \begin{cases}c_{-}, & \text {for } j<0 \\ u_{\chi}, & \text { for } j=0 \\ c_{+}, & \text {for } j>0\end{cases}
$$

The following discrete entropy inequality is readily derived from (5.1):

$$
\left|U_{j}^{n+1}-C_{j}\right| \leq\left|U_{j}^{n}-C_{j}\right|-\lambda \Delta_{-} H_{j+\frac{1}{2}}+\lambda\left|\Delta_{-} h\left(x_{j+\frac{1}{2}}, C_{j+1}, C_{j}\right)\right| .
$$

We multiply the cell entropy inequality (5.8) by $\phi_{j}^{n} \Delta x$, and then sum by parts to get

$$
\begin{aligned}
& \underbrace{\Delta x \Delta t \sum_{n \geq 0} \sum_{j \in \mathbf{Z}}\left|U_{j}^{n+1}-C_{j}\right|\left(\phi_{j}^{n+1}-\phi_{j}^{n}\right) / \Delta t}_{\mathcal{S}_{1}} \\
& +\underbrace{\Delta x \Delta t \sum_{n \geq 0} \sum_{j \in \mathbf{Z}} H_{j-\frac{1}{2}}^{n} \cdot\left(\Delta_{-} \phi_{j}^{n} / \Delta x\right)}_{\mathcal{S}_{2}}+\underbrace{\Delta x \Delta t \sum_{n \geq 0} \sum_{j \in \mathbf{Z}}\left(\left|\Delta_{-} h\left(x_{j+\frac{1}{2}}, C_{j+1}, C_{j}\right)\right| / \Delta x\right) \phi_{j}^{n} \geq 0 .}_{\mathcal{S}_{3}} .
\end{aligned}
$$

By Lebesgue's dominated convergence theorem,

$$
\mathcal{S}_{1} \rightarrow \iint_{\Pi_{T}}|u-\alpha| \phi_{t} d t d x \text { as } \Delta \rightarrow 0
$$

For $\mathcal{S}_{2}$ and $\mathcal{S}_{3}$, we consider two cases.

Case $1\left(\alpha(x)\right.$ has a jump, $\left.c_{-} \neq c_{+}\right)$. We first address $\mathcal{S}_{2}$. From (4.4), (5.2), and (5.7) we find that

$$
H_{j-\frac{1}{2}}^{n}= \begin{cases}\bar{g}\left(U_{j}^{n} \vee c_{-}, U_{j-1}^{n} \vee c_{-}\right)-\bar{g}\left(U_{j}^{n} \wedge c_{-}, U_{j-1}^{n} \wedge c_{-}\right), & j<0, \\ \bar{g}\left(U_{0}^{n} \vee u_{\chi}, U_{-1}^{n} \vee c_{-}\right)-\bar{g}\left(U_{0}^{n} \wedge u_{\chi}, U_{-1}^{n} \wedge c_{-}\right), & j=0, \\ \bar{f}\left(U_{1}^{n} \vee c_{+}, U_{0}^{n} \vee u_{\chi}\right)-\bar{f}\left(U_{1}^{n} \wedge c_{+}, U_{0}^{n} \wedge u_{\chi}\right), & j=1, \\ \bar{f}\left(U_{j}^{n} \vee c_{+}, U_{j-1}^{n} \vee c_{+}\right)-\bar{f}\left(U_{j}^{n} \wedge c_{+}, U_{j-1}^{n} \wedge c_{+}\right), & j>1 .\end{cases}
$$

We break up $\mathcal{S}_{2}$ using 5.10 :

$$
\mathcal{S}_{2}=\left(\Delta x \Delta t \sum_{n \geq 0} \sum_{j<0}+\Delta x \Delta t \sum_{n \geq 0} \sum_{j=0}^{1}+\Delta x \Delta t \sum_{n \geq 0} \sum_{j>1}\right) H_{j-\frac{1}{2}}^{n} \cdot\left(\Delta_{-} \phi_{j}^{n} / \Delta x\right) .
$$

The middle sum vanishes in the limit as $\Delta \rightarrow 0$, and the first and third sums converge by standard arguments (cf. [18, 38]) to

$$
\int_{0}^{T} \int_{x<0} \operatorname{sign}\left(u-c_{-}\right)\left(g(u)-g\left(c_{-}\right)\right) \phi_{x} d x d t \text { and } \int_{0}^{T} \int_{x>0} \operatorname{sign}\left(u-c_{+}\right)\left(f(u)-f\left(c_{+}\right)\right) \phi_{x} d x d t .
$$

Recalling the definition of $\mathcal{F}(x, \cdot)$, we see by adding the two integrals in (5.11) that

$$
\mathcal{S}_{2} \rightarrow \iint_{\Pi_{T}} \operatorname{sign}(u-\alpha)(\mathcal{F}(x, u)-\mathcal{F}(x, \alpha)) \phi_{x} d t d x
$$


Next, we claim that

$$
\mathcal{S}_{3} \rightarrow \int_{0}^{T}|f(\alpha(0+))-g(\alpha(0-))| \phi(0, t) d t .
$$

Using the definition of $C_{j}$, along with the consistency of the Godunov flux, we can write the sum $\mathcal{S}_{3}$ as follows:

$$
\begin{aligned}
\mathcal{S}_{3}= & \Delta x \Delta t \sum_{n=0}^{N} \sum_{|j| \leq J}\left(\left|\Delta_{-} h\left(x_{j+\frac{1}{2}}, C_{j+1}, C_{j}\right)\right| / \Delta x\right) \phi_{j}^{n} \\
= & \Delta \sum_{n=0}^{N} \phi_{0}^{n}\left|h\left(x_{\frac{1}{2}}, c_{+}, u_{\chi}\right)-h\left(x_{-\frac{1}{2}}, u_{\chi}, c_{-}\right)\right| \\
& +\Delta t \sum_{n=0}^{N} \phi_{1}^{n}\left|f\left(c_{+}\right)-h\left(x_{\frac{1}{2}}, c_{+}, u_{\chi}\right)\right| \\
& +\Delta t \sum_{n=0}^{N} \phi_{-1}^{n}\left|h\left(x_{-\frac{1}{2}}, u_{\chi}, c_{-}\right)-g\left(c_{-}\right)\right| \\
& +\Delta x \Delta t \sum_{n=0}^{N} \sum_{j=-J}^{-2} \phi_{j}^{n}\left|\frac{1}{\Delta x} \Delta_{+} g\left(c_{-}\right)\right|+\Delta x \Delta t \sum_{n=0}^{N} \sum_{j=2}^{J} \phi_{j}^{n}\left|\frac{1}{\Delta x} \Delta_{+} f\left(c_{+}\right)\right| .
\end{aligned}
$$

Clearly, the last line of 5.14 vanishes. Due to 5.7 and 2.1 we have

$$
h\left(x_{\frac{1}{2}}, c_{+}, u_{\chi}\right)=f\left(c_{+}\right), \quad h\left(x_{-\frac{1}{2}}, u_{\chi}, c_{-}\right)=g\left(c_{-}\right),
$$

and so the second and third sums vanish, leaving us with

$$
\mathcal{S}_{3}=\Delta t \sum_{n=0}^{N} \phi_{0}^{n}\left|f\left(c_{+}\right)-g\left(c_{-}\right)\right|,
$$

making it clear that the claim $(5.13)$ is true. Combining $5.9,5$ (5.12) and 5.13 completes the proof of Condition (D.4) for Case 1.

Case $2(\alpha(x)$ is constant, i.e., $\alpha(x)=c$ for all $x \in \mathbb{R})$. In this case, formula $(5.10)$ simplifies to

$$
H_{j-\frac{1}{2}}^{n}= \begin{cases}\bar{g}\left(U_{j}^{n} \vee c, U_{j-1}^{n} \vee c\right)-\bar{g}\left(U_{j}^{n} \wedge c, U_{j-1}^{n} \wedge c\right), & j \leq 0, \\ \bar{f}\left(U_{j}^{n} \vee c, U_{j-1}^{n} \vee c\right)-\bar{f}\left(U_{j}^{n} \wedge c, U_{j-1}^{n} \wedge c\right), & j \geq 1,\end{cases}
$$

from which it is clear that 5.12 holds for $\mathcal{S}_{2}$ in this situation also.

For $\mathcal{S}_{3}$, formula (5.14) simplifies to

$$
\begin{aligned}
\mathcal{S}_{3}=\Delta & t \sum_{n=0}^{N} \phi_{0}^{n}|f(c)-g(c)| \\
& \quad+\Delta x \Delta t \sum_{n=0}^{N} \sum_{j=-J}^{-2} \phi_{j}^{n}\left|\frac{1}{\Delta x} \Delta_{+} g(c)\right|+\Delta x \Delta t \sum_{n=0}^{N} \sum_{j=2}^{J} \phi_{j}^{n}\left|\frac{1}{\Delta x} \Delta_{+} f(c)\right| \\
=\Delta & t \sum_{n=0}^{N} \phi_{0}^{n}|f(c)-g(c)| .
\end{aligned}
$$

It is now clear that

$$
\mathcal{S}_{3} \rightarrow \int_{0}^{T}|f(c)-g(c)| \phi(0, t) d t
$$

Combining (5.9), 5.12) and (5.15) we see that Condition (D.4) holds for Case 2.

We can now state and prove our main theorem. 
Theorem 5.1 (Convergence). Assume that $(1.2),(1.3),(1.4), \sqrt{1.5}$ and $(1.6)$ hold. Let $u^{\Delta}$ be defined by the scheme of Section 4. Let $\Delta \rightarrow 0$ with $\lambda=\Delta x / \Delta t$ constant and the CFL condition (4.1) satisfied. Let $u(x, t)$ denote the unique vanishing viscosity solution of (1.1). Then $u^{\Delta} \rightarrow u$ in $L_{\text {loc }}^{1}\left(\Pi_{T}\right)$ and a.e. in $\Pi_{T}$.

Proof. Exactly as in the proof of Theorem 5.1 of [19, it follows from Lemma 5.1 that there is a subsequence $u^{\Delta}$ (which we do not bother to relabel) such that $u^{\Delta}$ converges in $L_{\text {loc }}^{1}\left(\Pi_{T}\right)$ and also boundedly a.e. in $\Pi_{T}$ to some $u \in L^{\infty}\left(\Pi_{T}\right)$ with $u(x, t) \in[\underline{u}, \bar{u}]$ for a.e. $(x, t) \in \Pi_{T}$.

By Lemma 5.3. the limit $u$ is an entropy solution of type $\mathcal{V}$. By Theorem 3.2 , it is thus also the vanishing viscosity solution. By the Theorem 3.1 (the uniqueness theorem), the entire computed sequence $u^{\Delta}$ (not just a subsequence) converges to $u$ in $L_{\text {loc }}^{1}\left(\Pi_{T}\right)$ and a.e. in $\Pi_{T}$.

As a consequence of the preceding theorem, we have the following result, stating that the two solution concepts, entropy solution of type $\mathcal{V}$ and vanishing viscosity solution, are equivalent.

Theorem 5.2. With the assumptions (1.2), (1.3), 1.4), 1.5) and (1.6), a measurable function $u: \Pi_{T} \rightarrow \mathbb{R}$ is an entropy solution of type $\mathcal{V}$ if and only if it is a vanishing viscosity solution.

Proof. By Theorem 3.2, we only have to show that a vanishing viscosity solution is an entropy solution of type $\mathcal{V}$. So, assume the measurable function $u: \Pi_{T} \rightarrow \mathbb{R}$ is the vanishing viscosity solution of the Cauchy problem (1.1). By Theorem 5.1 $u$ is the limit of approximations generated by the scheme of Section 4 . By Lemma 5.3, $u$ is an entropy solution of type $\mathcal{V}$.

\section{Characteristics at the interface}

Lemma 6.1. Let $u$ be a vanishing viscosity solution. Let $t \in(0, T)$ be a time where the traces $u_{-}$ and $u_{+}$exist. Then the pair $\left(u_{-}, u_{+}\right)$satisfies the following weak characteristic condition:

$$
\text { if } u_{-} \neq u_{+} \text {, then } \min \left(0, g_{u}\left(u_{-}\right)\right) \cdot \max \left(0, f_{u}\left(u_{+}\right)\right)=0 \text {. }
$$

Remark 6.1. Condition 6.1 requires that the characteristics on at least one side of the interface can be traced backwards in time to the $x$-axis. (Recall that in the classical case of a scalar onedimensional conservation law, the Lax condition requires that the characteristics on both sides of a shock can be traced back to the $x$-axis.) If the solution happens to be continuous at the interface $\left(u_{-}=u_{+}\right)$, there is no restriction on the characteristics, and undercompressive jumps are allowed. In [19] the condition [6.1] is referred to as the weak characteristic condition, and the following as the strong characteristic condition:

$$
\min \left(0, g_{u}\left(u_{-}\right)\right) \cdot \max \left(0, f_{u}\left(u_{+}\right)\right)=0 .
$$

The strong characteristic condition rules out undercompressive jumps in all cases. See Remark 3.2 .

Proof. Take the case where $u_{+}<u_{-}$. The proof of the case where $u_{+}>u_{-}$is similar and we omit it. By way of obtaining a contradiction, suppose that

$$
g_{u}\left(u_{-}\right)<0, \quad f_{u}\left(u_{+}\right)>0 .
$$

By Theorem 5.2, it suffices to obtain a contradiction to the $\Gamma_{1}$ condition. By $(6.2), g$ is strictly decreasing near $u_{-}$, and $f$ is strictly increasing near $u_{+}$. Thus, there are points $v$ and $w$ such that

$$
u_{+}<v<w<u_{-}, \quad f(v)>f\left(u_{+}\right), \quad g(w)>g\left(u_{-}\right) .
$$

First, assume that there are no flux crossings in the interval $\left(u_{+}, u_{-}\right)$. Then either

(1) $f(z) \geq g(z)$ for $z \in\left[u_{+}, u_{-}\right]$, or

(2) $f(z) \leq g(z)$ for $z \in\left[u_{+}, u_{-}\right]$.

In Case (1), 6.3 implies $f(w) \geq g(w)>g\left(u_{-}\right)$. In Case (2), 6.3 implies $f\left(u_{+}\right)<f(v) \leq g(v)$. In either case, Condition B1 of the $\Gamma_{1}$ condition is violated, and we have a contradiction.

Next, assume that there is at least one flux crossing in the interval $\left(u_{+}, u_{-}\right)$. By moving $v$ closer to $u_{+}$and $w$ closer to $u_{-}$if necessary, we may assume that there is at least one crossing, call it $u_{\chi}$, in the interval $(v, w)$, implying that $v \in\left[u_{+}, u_{\chi}\right], w \in\left[u_{\chi}, u_{-}\right]$. Then the inequalities $f(v)>f\left(u_{+}\right), g(w)>g\left(u_{-}\right)$of $(6.3)$ violate Condition B2 of the $\Gamma_{1}$ condition, again giving us the desired contradiction. 

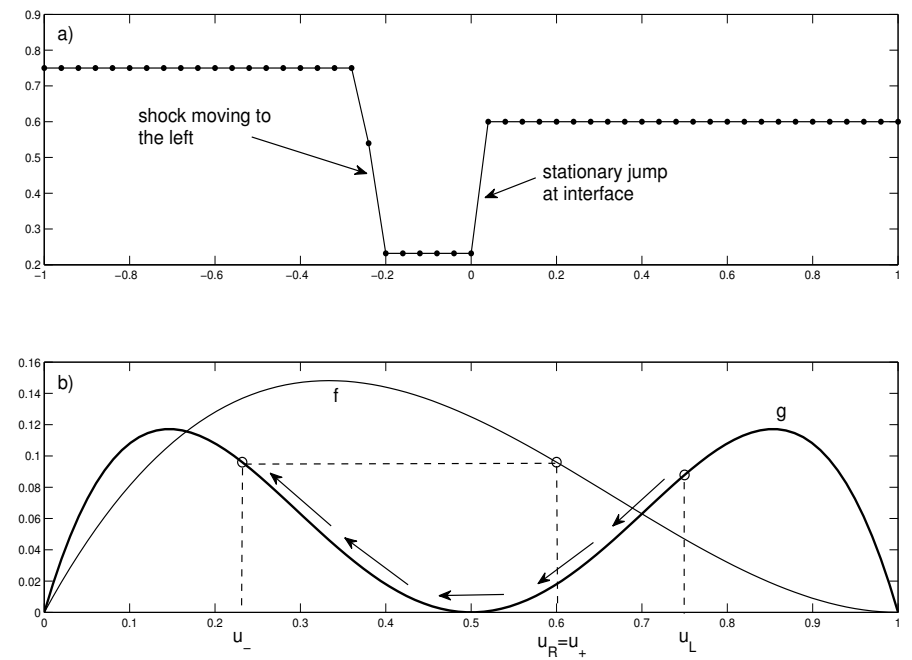

Figure 4. Example 1. $\Delta x=.04, \Delta t=.02,800$ time steps. The pair $\left(u_{-}, u_{+}\right)$ satisfies the $\Gamma$ condition with $\hat{u}=u_{-}$.

\section{NUMERICAL EXAMPLES}

We present some numerical examples, all of which are Riemann problems. The initial data has the form $u_{0}(x)=H(-x) u_{L}+H(x) u_{R}$, and formula 4.2 yields the discretization

$$
U_{j}^{0}= \begin{cases}u_{L}, & j<0, \\ \left(u_{L}+u_{R}\right) / 2, & j=0, \\ u_{R}, & j>0 .\end{cases}
$$

In this section we will use the notation

$$
u_{-}:=u(0-, t), \quad u_{+}:=u(0+, t)
$$

for the left and right traces of the solutions.

Example 1. The data for this Riemann problem is

$$
\begin{aligned}
& g(u)=7.5 u(1-u)(u-1 / 2)^{2}, \quad f(u)=u(1-u)^{2}, \\
& u_{L}=.75, \quad u_{R}=.6 .
\end{aligned}
$$

See Figure 4. There is a single flux crossing between the initial states $u_{L}$ and $u_{R}$. The solution develops a shock moving to the left, along with a stationary jump located at the flux interface $x=0$. The pair of states $\left(u_{-}, u_{+}\right)$satisfy the $\Gamma$ condition with $\hat{u}=u_{-}$.

Example 2. The fluxes $g$ and $f$ are the same as in Example 1. The initial data for this Riemann problem is

$$
u_{L}=.95, \quad u_{R}=.05 .
$$

See Figure 5. The solution consists of a left-facing rarefaction wave, a right-facing rarefaction wave, and a stationary jump at $x=0$. The pair $\left(u_{-}, u_{+}\right)$satisfies the $\Gamma$ condition with $\hat{u}=u_{+}$. When we discretize the initial data using (7.1), a spurious traveling wave, or bump, is created during the first few time steps, see plot a). It travels to the right, and spreads out. By refining the grid, one can verify that there is nevertheless convergence in $L_{\mathrm{loc}}^{1}$ and boundedly a.e., as guaranteed by Theorem 5.1 . 

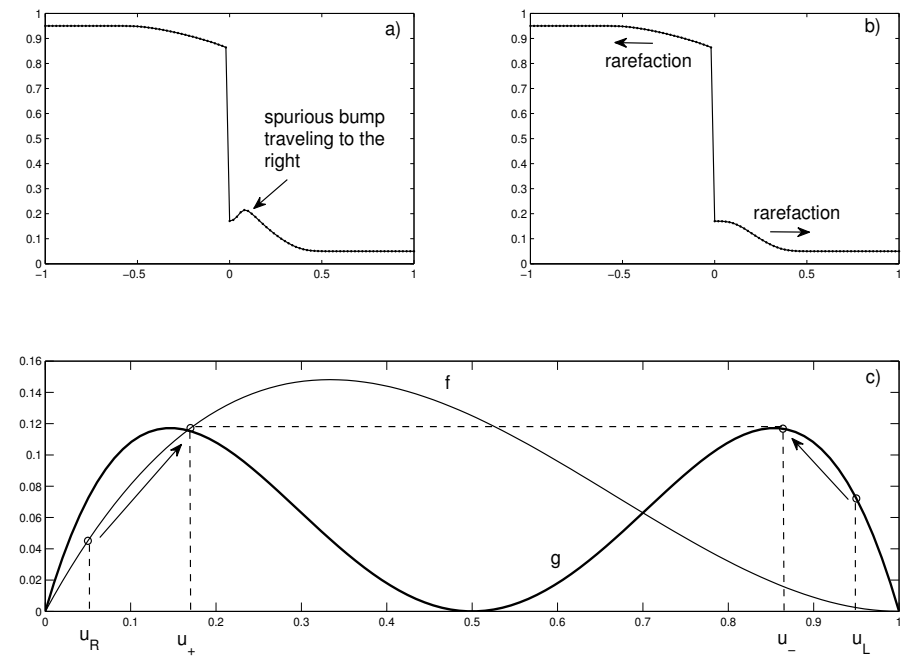

Figure 5. Example 2. $\Delta x=.02, \Delta t=.01,40$ time steps. Plot a) uses the discretization (7.1). Plot b) uses the discretization (7.2), and gets rid of the spurious bump. The pair $\left(u_{-}, u_{+}\right)$satisfies the $\Gamma$ condition with $\hat{u}=u_{+}$.

By shifting the initial data $u_{0}(x)$ by a half mesh width, it is possible to get rid of the bump. To produce plot b), we first shifted the initial data to the left by $\Delta x / 2$, and then applied (4.2), which results in

$$
U_{j}^{0}= \begin{cases}u_{L}, & j<0, \\ u_{R}, & j \geq 0 .\end{cases}
$$

Based on a moderate amount of numerical experience with Riemann problems, it seems that if a spurious bump appears, it is always possible to get rid of it via either a left or right shift of $\Delta x / 2$. This type of shift does not affect the convergence result because for Theorem 5.1 we only require that

$$
\sum_{j \in \mathbb{Z}} U_{j}^{0} \chi_{j}(x) \rightarrow u_{0}(x) \text { in } L_{\mathrm{loc}}^{1} \text { and boundedly a.e. }
$$

Example 3. For this example, we swapped $f$ and $g$. The data for this example is:

$$
\begin{aligned}
& g(u)=u(1-u)^{2}, \quad f(u)=7.5 u(1-u)(u-1 / 2)^{2}, \\
& u_{L}=.05, \quad u_{R}=.95 .
\end{aligned}
$$

See Figure 6. There are two flux crossings between the initial states $u_{L}$ and $u_{R}$. The solution consists of a shock moving to the right and a stationary jump at $x=0$. The pair $\left(u_{-}, u_{+}\right)$satisfies the $\Gamma$ condition with $\hat{u}=u_{+}$.

Example 4. For this example, $f$ and $g$ are the same as in Example 3. The initial data is

$$
u_{L}=.1, \quad u_{R}=.9 .
$$

See Figure 7. The solution has a shock moving to the left, a shock moving to the right, and there is no jump at the interface. What is interesting about this example is that the solution is continuous across the interface: $u_{-}=u_{+}=u_{\chi}$. Also, the solution is undercompressive at the interface, i.e., $g^{\prime}\left(u_{-}\right)<0<f^{\prime}\left(u_{+}\right)$. See Remark 3.2. In this example the pair $\left(u_{-}, u_{+}\right)$satisfies the $\Gamma$ condition trivially with $\hat{u}=u_{-}=u_{+}=u_{\chi}$. 

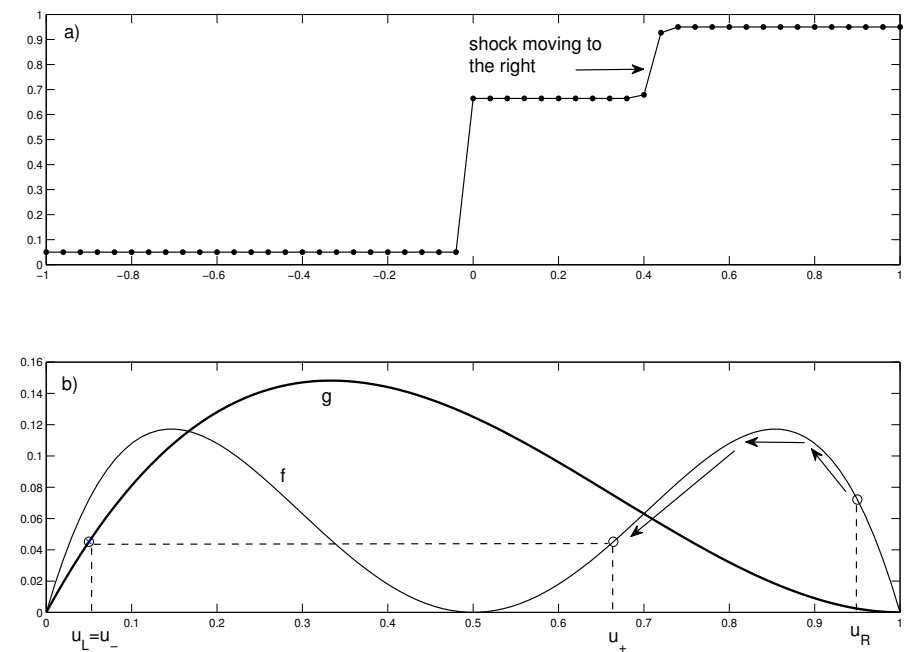

Figure 6. Example 3. $\Delta x=.04, \Delta t=.02,200$ time steps. The pair $\left(u_{-}, u_{+}\right)$ satisfies the $\Gamma$ condition with $\hat{u}=u_{+}$.
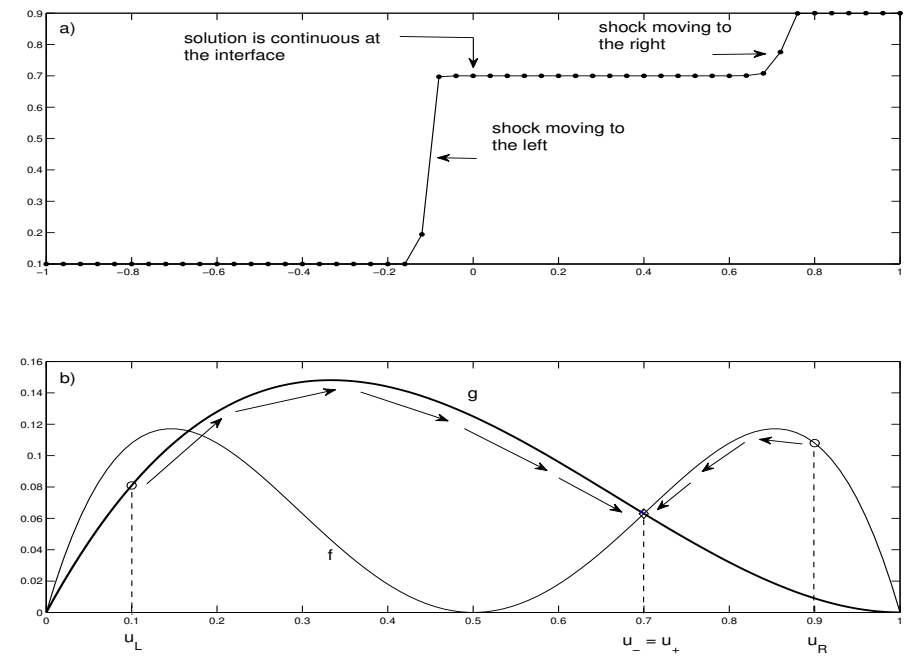

Figure 7. Example 4. $\Delta x=.04, \Delta t=.02,150$ time steps. The solution is continuous across the interface, with $u_{-}=u_{+}=u_{\chi}$. The pair $\left(u_{-}, u_{+}\right)$satisfies the $\Gamma$ condition with $\hat{u}=u_{-}=u_{+}=u_{\chi}$.

\section{REFERENCES}

[1] Adimurthi, J. Jaffré, and G. D. Veerappa Gowda. Godunov-type methods for conservation laws with a flux function discontinuous in space. SIAM J. Numer. Anal., 42(1):179-208 (electronic), 2004.

[2] Adimurthi, S. Mishra, and G. D. V. Gowda. Optimal entropy solutions for conservation laws with discontinuous flux-functions. J. Hyperbolic Differ. Equ., 2(4):783-837, 2005.

[3] Adimurthi, S. Mishra, and G. D. V. Gowda. Existence and stability of entropy solutions for a conservation law with discontinuous non-convex fluxes. Netw. Heterog. Media, 2(1):127-157 (electronic), 2007.

[4] Adimurthi, R. Dutta, G. D. V. Gowda, and J. Jaffré. Monotone $(A, B)$ entropy stable numerical scheme for scalar conservation laws with discontinuous flux. ESAIM Math. Model. Numer. Anal., 48(6):1725-1755, 2014. 
[5] Adimurthi, K. Sudarshan Kumar, and G. D. Veerappa Gowda. Second order scheme for scalar conservation laws with discontinuous flux. Appl. Numer. Math., 80:46-64, 2014.

[6] B. Andreianov, K. H. Karlsen, and N. H. Risebro. A theory of $L^{1}$-dissipative solvers for scalar conservation laws with discontinuous flux. Arch. Ration. Mech. Anal., 201(1):27-86, 2011.

[7] B. Andreianov, K. H. Karlsen, and N. H. Risebro. On vanishing viscosity approximation of conservation laws with discontinuous flux. Netw. Heterog. Media, 5(3):617-633, 2010.

[8] B. Andreianov and C. Cancès. The Godunov scheme for scalar conservation laws with discontinuous bell-shaped flux functions. Appl. Math. Lett., 25(11):1844-1848, 2012.

[9] B. Andreianov and C. Cancès. Vanishing capillarity solutions of Buckley-Leverett equation with gravity in two-rocks' medium. Comput. Geosci., 17(3):551-572, 2013.

[10] B. Andreianov and C. Cancès. On interface transmission conditions for conservation laws with discontinuous flux of general shape. J. Hyperbolic Differ. Equ., 12(2):343-384, 2015.

[11] B. Andreianov and D. Mitrović. Entropy conditions for scalar conservation laws with discontinuous flux revisited. Ann. Inst. H. Poincaré Anal. Non Linéaire, 32(6):1307-1335, 2015.

[12] E. Audusse and B. Perthame. Uniqueness for scalar conservation laws with discontinuous flux via adapted entropies. Proc. Roy. Soc. Edinburgh Sect. A, 135(2):253-265, 2005.

[13] F. Bachmann and J. Vovelle. Existence and uniqueness of entropy solution of scalar conservation laws with a flux function involving discontinuous coefficients. Comm. Partial Differential Equations, 31(1-3):371-395, 2006.

[14] P. Baiti and H. K. Jenssen. Well-posedness for a class of $2 \times 2$ conservation laws with $L^{\infty}$ data. J. Differential Equations, 140(1):161-185, 1997.

[15] R. Bürger, K. H. Karlsen, C. Klingenberg, and N. H. Risebro. A front tracking approach to a model of continuous sedimentation in ideal clarifier-thickener units. Nonlinear Anal. Real World Appl., 4(3):457-481, 2003

[16] R. Bürger, A. García, K. H. Karlsen, and J. D. Towers. On an extended clarifier-thickener model with singular source and sink terms. European J. Appl. Math., 17:257-292, 2006.

[17] R. Bürger, K. H. Karlsen, N. H. Risebro, and J. D. Towers. Well-posedness in $B V_{t}$ and convergence of a difference scheme for continuous sedimentation in ideal clarifier-thickener units. Numer. Math., 97(1):25-65, 2004.

[18] R. Bürger, A. García, K. H. Karlsen, and J. D. Towers. A family of numerical schemes for kinematic flows with discontinuous flux. J. Engrg. Math., 60:387-425, 2008.

[19] R. Bürger, K. H. Karlsen, and J. D. Towers. An Engquist-Osher-type scheme for conservation laws with discontinuous flux adapted to flux connections. SIAM J. Numer. Anal., 47(3):1684-1712, 2009.

[20] R. Bürger, K. H. Karlsen, H. Torres, and J. D. Towers. Second-order schemes for conservation laws with discontinuous flux modelling clarifier-thickener units. Numer. Math., 116(4):579 - 617, 2010.

[21] R. Bürger, K. H. Karlsen, and J. D. Towers. A model of continuous sedimentation of flocculated suspensions in clarifier-thickener units. SIAM J. Appl. Math., 65(3):882-940 (electronic), 2005.

[22] G.-Q. Chen, N. Even, and C. Klingenberg. Hyperbolic conservation laws with discontinuous fluxes and hydrodynamic limit for particle systems. J. Differential Equations, 245(11):3095-3126, 2008.

[23] M. G. Crandall and A. Majda. Monotone difference approximations for scalar conservation laws. Math. Comp., 34(149):1-21, 1980.

[24] G. Crasta, V. De Cicco, G. De Philippis, and F. Ghiraldin. Structure of solutions of multidimensional conservation laws with discontinuous flux and applications to uniqueness. Arch. Ration. Mech. Anal., 221(2):961-985, 2016.

[25] G. Crasta, V. De Cicco, and G. De Philippis. Kinetic formulation and uniqueness for scalar conservation laws with discontinuous flux. Comm. Partial Differential Equations, 40(4):694-726, 2015.

[26] S. Diehl. On scalar conservation laws with point source and discontinuous flux function. SIAM J. Math. Anal., 26(6):1425-1451, 1995.

[27] S. Diehl. Scalar conservation laws with discontinuous flux function. I. The viscous profile condition. Comm. Math. Phys., 176(1):23-44, 1996.

[28] S. Diehl. A conservation law with point source and discontinuous flux function modelling continuous sedimentation. SIAM J. Appl. Math., 56(2):388-419, 1996.

[29] S. Diehl. A uniqueness condition for nonlinear convection-diffusion equations with discontinuous coefficients. J. Hyperbolic Differ. Equ., 6(1):127-159, 2009.

[30] M. Garavello, R. Natalini, B. Piccoli, and A. Terracina. Conservation laws with discontinuous flux. Netw. Heterog. Media, 2:159-179, 2007.

[31] T. Gimse and N. H. Risebro. Riemann problems with a discontinuous flux function. In Proc. 3rd Internat. Conf. Hyperbolic Problems, pages 488-502, Uppsala, 1991. Studentlitteratur.

[32] T. Gimse and N. H. Risebro. Solution of the Cauchy problem for a conservation law with a discontinuous flux function. SIAM J. Math. Anal., 23(3):635-648, 1992.

[33] H. Holden and N. H. Risebro. Front tracking for hyperbolic conservation laws, volume 152 of Applied Mathematical Sciences. Springer-Verlag, New York, 2002.

[34] J. Jimenez. Mathematical analysis of a scalar multidimensional conservation law with discontinuous flux Journal of Evolution Equations, 11(3):553-576, 2011. 
[35] K. H. Karlsen and N. H. Risebro. Convergence of finite difference schemes for viscous and inviscid conservation laws with rough coefficients. M2AN Math. Model. Numer. Anal., 35(2):239-269, 2001.

[36] K. H. Karlsen, N. H. Risebro, and J. D. Towers. On a nonlinear degenerate parabolic transport-diffusion equation with a discontinuous coefficient. Electron. J. Differential Equations, pages No. 93, 23 pp. (electronic), 2002.

[37] K. H. Karlsen, N. H. Risebro, and J. D. Towers. Upwind difference approximations for degenerate parabolic convection-diffusion equations with a discontinuous coefficient. IMA J. Numer. Anal., 22(4):623-664, 2002.

[38] K. H. Karlsen, N. H. Risebro, and J. D. Towers. $L^{1}$ stability for entropy solutions of nonlinear degenerate parabolic convection-diffusion equations with discontinuous coefficients. Skr. K. Nor. Vidensk. Selsk., (3):149, 2003.

[39] C. Klingenberg and N. H. Risebro. Convex conservation laws with discontinuous coefficients. Existence, uniqueness and asymptotic behavior. Comm. Partial Differential Equations, 20(11-12):1959-1990, 1995

[40] R. J. LeVeque. Numerical methods for conservation laws. Lectures in Mathematics ETH Zürich. Birkhäuser Verlag, Basel, second edition, 1992

[41] S. Mishra. Convergence of upwind finite difference schemes for a scalar conservation law with indefinite discontinuities in the flux function. SIAM J. Numer. Anal., 43(2):559-577 (electronic), 2005.

[42] D. Mitrovic. New entropy conditions for scalar conservation laws with discontinuous flux. Discrete Contin. Dyn. Syst., 30(4):1191-1210, 2011.

[43] E. Y. Panov. On existence and uniqueness of entropy solutions to the cauchy problem for a conservation law with discontinuous flux. J. Hyperbolic Differ. Equ., 06(03):525-548, 2009.

[44] N. H. Risebro. An introduction to the theory of scalar conservation laws with spatially discontinuous flux functions. In Applied Wave Mathematics. Springer Verlag, to appear.

[45] N. Seguin and J. Vovelle. Analysis and approximation of a scalar conservation law with a flux function with discontinuous coefficients. Math. Models Methods Appl. Sci., 13(2):221-257, 2003.

[46] J. D. Towers. Convergence of a difference scheme for conservation laws with a discontinuous flux. SIAM J. Numer. Anal., 38(2):681-698 (electronic), 2000.

[47] J. D. Towers. A difference scheme for conservation laws with a discontinuous flux: the nonconvex case. SIAM J. Numer. Anal., 39(4):1197-1218 (electronic), 2001. 